\title{
SMOOTHNESS CRITERION FOR NAVIER-STOKES EQUATIONS IN TERMS OF REGULARITY ALONG THE STREAMLINES*
}

\author{
CHI HIN $\mathrm{CHAN}^{\dagger}$
}

\begin{abstract}
This article is devoted to a regularity criterion for solutions to the Navier-Stokes equations in terms of regularity along the streamlines. More precisely, we prove that if $u$ is a suitable weak solution to the Navier-Stokes equations on $[0, T] \times \mathbb{R}^{3}$ satisfying the condition that $\frac{|u \cdot \nabla F|}{|u| \gamma} \leqslant A|F|$, in which $F=\operatorname{div}\left(\frac{u}{|u|}\right), A$ is some given constant, and $\gamma$ is some positive number with $0<\gamma<\frac{1}{3}$, then it follows that $u$ is smooth over $(0, T] \times \mathbb{R}^{3}$.
\end{abstract}

Key words. Navier-Stokes equation, regularity criterion.

AMS subject classifications. 35B65, 76D03, 76D05

1. Introduction. In this article, we consider the Navier-Stokes equation on $\mathbb{R}^{3}$, given by

$$
\begin{aligned}
\partial_{t} u-\triangle u+\operatorname{div}(u \otimes u)+\nabla P & =0 \\
\operatorname{div}(u) & =0
\end{aligned}
$$

in which $u$ is a vector-valued function representing the velocity of the fluid, and $P$ is the pressure. Note that the pressure depends in a non local way on the velocity $u$. It can be seen as a Lagrange multiplier associated to the incompressible condition $\operatorname{div}(u)=$ 0 . The initial value problem of the above equations is endowed with the condition that $u(0, \cdot)=u_{0} \in L^{2}\left(\mathbb{R}^{3}\right)$. Leray [12] and Hopf [8] had already established the existence of global weak solutions to the Navier-Stokes equations. In particular, Leray introduced a notion of weak solutions for the Navier-Stokes equation, and proved that, for every given initial datum $u_{0} \in L^{2}\left(\mathbb{R}^{3}\right)$, there exists a global weak solution $u \in L^{\infty}\left(0, \infty ; L^{2}\left(\mathbb{R}^{3}\right)\right) \cap L^{2}\left(0, \infty ; \dot{H}^{1}\left(\mathbb{R}^{3}\right)\right)$ verifying the Navier-Stokes equation in the sense of distribution. From that time on, much effort has been devoted to establish the global existence and uniqueness of smooth solutions to the Navier-Stokes equations. Different Criteria for regularity of the weak solutions have been proposed. The ProdiSerrin condition (see Serrin [16], Prodi [14], and [17]) states that any weak Leray-Hopf solution verifying $u \in L^{p}\left(0, \infty ; L^{q}\left(\mathbb{R}^{3}\right)\right)$ with $2 / p+3 / q=1,2 \leq p<\infty$, is regular on $(0, \infty) \times \mathbb{R}^{3}$. The limit case of $L^{\infty}\left(0, \infty ; L^{3}\left(\mathbb{R}^{3}\right)\right)$ has been solved very recently by $L$. Escauriaza, G. Seregin, and V. Sverak (see [7]) (see also the work [21] of Y. Zhou, in which a regularity criterion is obtained with a type of Prodi-Serrin condition to be imposed on only one velocity component). Here, we just mention a piece of work [4] by Ch.-H. Chan and A. Vasseur which is devoted to a log improvement of the ProdiSerrin criterion in the case of $p=q=5$. Other criteria have been later introduced, dealing with some derivatives of the velocity. Beale, Kato and Majda [1] showed the global regularity of solutions under the condition that the vorticity $\omega=\operatorname{curl} u$ lies in $L^{\infty}\left(0, \infty ; L^{1}\left(\mathbb{R}^{3}\right)\right)$ (see Kozono and Taniuchi for improvement of this result [10]). Beirão da Veiga proved in [2] that the boundedness of $\nabla u$ in $L^{p}\left(0, \infty ; L^{q}\left(\mathbb{R}^{3}\right)\right)$ for $2 / p+3 / q=2,1<p<\infty$ ensures the global regularity (see also [22], [11], and [23]). In [5], Constantin and Fefferman gave a regularity criterion with a condition

\footnotetext{
*Received December 8, 2009; accepted for publication April 23, 2010.

${ }^{\dagger}$ Institute for Mathematics and its Applications, University of Minnesota, 207 Church Street SE, Minneapolis, MN 55455-0134, USA (chana002@ima.umn.edu).
} 
involving only the direction of the vorticity (For further works along this direction, see, for instance, the works [24], and [25] of Y. Zhou and the references therein). Here, let us mention that there are also some regularity criteria established under some conditions to be imposed on the pressure term. For instance, in [15], G. Seregin and V. Sverak established a regularity criterion for solutions to the Navier-Stokes equations under the condition that the pressure term has a certain lower bound. On the other hand, regularity criteria in terms of Serrin-type conditions imposed on the gradient of the pressure are obtained in the works [26], [27] of Y. Zhou.

Until more recently, in a short paper [20], A. Vasseur gave another regularity criterion which states that any Leray-Hopf weak solution $u$ to the Navier-Stokes equations satisfying $\operatorname{div}\left(\frac{u}{|u|}\right) \in L^{p}\left(0, \infty ; L^{q}\left(\mathbb{R}^{3}\right)\right)$ with $\frac{2}{p}+\frac{3}{q} \leqslant \frac{1}{2}$ is necessary smooth on $(0, \infty) \times \mathbb{R}^{3}$. As we can see, the regularity criterion given in $[20]$ is the one with some integrable condition imposed on $\operatorname{div}\left(\frac{u}{|u|}\right)$. However, the goal of this paper is to obtain the full regularity of a suitable weak solution $u$ under some suitable assumption about the smoothness of $\operatorname{div}\left(\frac{u}{|u|}\right)$ along the streamlines of the fluid. More precisely, the goal of this paper is to prove the following theorem

THEOREM 1.1. Let $u$ be a suitable weak solution to the Navier-Stokes equation on $(0, T] \times \mathbb{R}^{3}$ which satisfies the condition that $\left|\frac{u \cdot \nabla F}{|u|^{\gamma}}\right| \leqslant A|F|$, in which $A$ is some positive constant, and $\gamma$ is some positive constant for which $0<\gamma<\frac{1}{3}$. Then, it follows that $u$ is a smooth solution on $(0, T] \times \mathbb{R}^{3}$.

As for Theorem 1.1, we note that $F=\operatorname{div}\left(\frac{u}{|u|}\right)$ can be rewritten as $F=-\frac{u \cdot \nabla|u|}{|u|^{2}}$, and hence is the derivative of $|u|$ along the streamlines of the fluid. Then, the condition appearing in the hypothesis of Theorem 1.1 can be seen as a constraint on the second derivative of $|u|$ along the streamlines. Theorem 1.1 itself shows that such a constraint on the second derivative of $|u|$ along the streamlines is enough to give the full regularity of the solution.

Before we proceed any further, let us say something about the term suitable weak solution. The concept of suitable weak solutions for Navier-Stokes equations was first introduced by Caffarelli, Kohn, and Nirenberg in [3] for the purpose of developing the partial regularity theory for solutions to the Navier-Stokes equations. By a suitable weak solution for the Navier-Stokes equations, we mean a Leray-Hopf weak solution $u \in L^{\infty}\left(0, T ; L^{2}\left(\mathbb{R}^{3}\right)\right) \cap L^{2}\left(0, T ; \dot{H}^{1}\left(\mathbb{R}^{3}\right)\right)$ which satisfies the following inequality in the sense of distribution on $(0, T) \times \mathbb{R}^{3}$.

$$
\partial_{t}\left(\frac{|u|^{2}}{2}\right)+\operatorname{div}\left(\frac{|u|^{2}}{2} u\right)+\operatorname{div}(P u)+|\nabla u|^{2}-\triangle\left(\frac{|u|^{2}}{2}\right) \leqslant 0 .
$$

Here, we decide to work with suitable weak solutions instead of just Leray-Hopf weak solutions because suitable weak solutions satisfy some very nice properties such as the partial regularity Theorem due to Caffarelli, Kohn, and Nirenberg in their joint work [3] (see also the related works of F. Lin [13], A. Vasseur [19], G. Tian and Z. Xin [18] ). Now, let us turn our attention back to Theorem 1.1. Indeed the conclusion of Theorem 1.1 will follow at once provided if we can prove the following proposition.

Proposition 1.2. Let $u$ be a suitable weak solution to the Navier-Stokes equa-

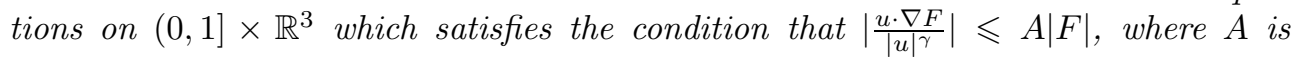
some positive constant, and $\gamma$ is some positive number satisfying $0<\gamma<\frac{1}{3}$. It then 
follows that $u$ is essentially bounded over the region $\left[\frac{3}{4}, 1\right] \times \mathbb{R}^{3}$. That is, we have $\|u\|_{L^{\infty}\left(\left[\frac{3}{4}, 1\right] \times \mathbb{R}^{3}\right)}<\infty$.

Before we devote our effort to prove proposition 1.2, let us first explain why proposition 1.2 will lead to the conclusion of Theorem 1.1 as follows. Assume that proposition 1.2 is indeed true. Without the loss of generality, let us assume that $u$ is a suitable weak solution to the Navier-Stokes equations on $(0,1] \times \mathbb{R}^{3}$ satisfying the hypothesis of Theorem 1.1 (we note that if our suitable weak solution $u$ is over $(0, T] \times \mathbb{R}^{3}$, with $T$ to be some positive number other than 1 , we can always rescale our weak solution $u$ ). Now, proposition 1.2 automatically tells us that $u$ is essentially bounded on the region $\left[\frac{3}{4}, 1\right] \times \mathbb{R}^{3}$. So, over such a region, we can apply the Serrin criterion with $p=q=\infty$ to conclude that $u$ is smooth over $\left(\frac{3}{4}, 1\right) \times \mathbb{R}^{3}$. So, the only question remains is how to justify that $u$ is also smooth over $\left(0, \frac{7}{8}\right) \times \mathbb{R}^{3}$. So, to finish our job, let $\tau \in\left(0, \frac{7}{8}\right)$ be arbritary chosen and fixed, and let us consider the function $u_{\lambda}(t, x)=\lambda u\left(\lambda^{2} t, \lambda x\right)$, with $\lambda=\left(\frac{8 \tau}{7}\right)^{\frac{1}{2}}$. Notice that $u_{\lambda}$ is then another suitable weak solution on $(0,1] \times \mathbb{R}^{3}$, which satisfies the same hypothesis of Theorem 1.1 (with a different constant $A_{\lambda}$, of course). So, we can invoke proposition 1.2 again to conclude that $u_{\lambda}$ is essentially bounded over $\left[\frac{3}{4}, 1\right] \times \mathbb{R}^{3}$. However, this means the same thing as saying that our original suitable weak solution $u$ is essentially bounded over the region $\left[\frac{6 \tau}{7}, \frac{8 \tau}{7}\right] \times \mathbb{R}^{3}$, and hence $u$ must be smooth over the region $\left(\frac{6 \tau}{7}, \frac{8 \tau}{7}\right) \times \mathbb{R}^{3}$. Since the number $\tau \in\left(0, \frac{7}{8}\right)$ is arbritary chosen in the above argument, we conclude that $u$ must be smooth over $(0,1) \times \mathbb{R}^{3}$, provided that proposition 1.2 is valid. So, it is clear that the main task of the whole paper is to prove proposition 1.2, which is what we will do in the following sections.

2. Basic setting of the whole paper. In order to prove proposition 1.2 , we would like to use the method of energy decompositions with respect to a sequence of cutting functions $v_{k}=\left\{|u|-R\left(1-\frac{1}{2^{k}}\right)\right\}_{+}$as introduced by A. Vasseur in [19]. Indeed, A. Vasseur was the first to use such a method of energy decompositions inherted from De Giorgi [6] to give a new proof of the famous Partial Regularity Theorem of Caffarelli, Kohn and Nirenberg (see [19]). So, we would like to introduce some notations first. Then, we will state one lemma and one proposition which are related to the proof of proposition 1.2. So, let us fix our notations as follow.

- for each $k \geqslant 0$, let $Q_{k}=\left[T_{k}, 1\right] \times \mathbb{R}^{3}$, in which $T_{k}=\frac{3}{4}-\frac{1}{4^{k+1}}$.

- for each $k \geqslant 0$, let $v_{k}=\left\{|u|-R\left(1-\frac{1}{2^{k}}\right)\right\}_{+}$.

- for each $k \geqslant 0$, let $d_{k}=\left.\frac{R\left(1-\frac{1}{2^{k}}\right)}{|u|} \chi_{\left\{|u|>R\left(1-\frac{1}{2^{k}}\right)\right\}}|\nabla| u\right|^{2}+\frac{v_{k}}{|u|}|\nabla u|^{2}$.

- for each $k \geqslant 0$, let $U_{k}=\frac{1}{2}\left\|v_{k}\right\|_{L^{\infty}\left(T_{k}, 1 ; L^{2}\left(\mathbb{R}^{3}\right)\right)}^{2}+\int_{T_{k}}^{1} \int_{\mathbb{R}^{3}} d_{k}^{2} d x d t$.

With the above setting, we are now ready to state the lemma and proposition which are related to proposition 1.2 as follow.

Proposition 2.1. Let $u$ be a suitable weak solution for the Navier-Stokes equation on $[0,1] \times \mathbb{R}^{3}$ which satisfies the condition that $\left|\frac{u \cdot \nabla F}{|u|^{\gamma}}\right| \leqslant A|F|$, where $A$ is some finite-positive constant, and $\gamma$ is some positive number satisfying $0<\gamma<\frac{1}{3}$. Then, there exists some constant $C_{p, \beta}$, depending only on $1<p<\frac{5}{4}$, and $\beta>\frac{6-3 p}{10-8 p}$, and also some constants $0<\alpha, K<\infty$, which do depend on our suitable weak solution $u$, such that the following inequality holds 


$$
\begin{aligned}
& U_{k} \leqslant C_{p, \beta} 2^{\frac{10 k}{3}}\left\{\frac{1}{R^{\beta \frac{10-8 p}{3 p}-\frac{2-p}{p}}}\|u\|_{L^{\infty}\left(0,1 ; L^{2}\left(\mathbb{R}^{3}\right)\right)}^{2\left(1-\frac{1}{p}\right)} U_{k-1}^{\frac{5-p}{3 p}}+\right. \\
& (1+A)\left(1+\frac{1}{\alpha}\right)\left(1+K^{1-\frac{1}{p}}\right)\left(1+\|u\|_{L^{\infty}\left(0,1 ; L^{2}\left(\mathbb{R}^{3}\right)\right)}\right) \times \\
& \left.\left[\left(\frac{1}{R^{\frac{10}{3}-2 p \beta+1-\gamma-p}}\right)^{\frac{1}{p}} U_{k-1}^{\frac{5}{3 p}}+\frac{1}{R^{\frac{10}{3}-2 \beta-\gamma}} U_{k-1}^{\frac{5}{3}}\right]\right\},
\end{aligned}
$$

for every sufficiently large $R>1$.

Here, let us make some important comments on the conclusion of proposition 2.1. As indicated by the inequality which appears in the conclusion of proposition 2.1, it is important for us to emphasis that those terms such as $R^{\beta \frac{10-8 p}{3 p}-\frac{2-p}{p}}, R^{\frac{10}{3}-2 p \beta+1-\gamma-p}$, and $R^{\frac{10}{3}-2 \beta-\gamma}$ should all appear in the denomerator. But unfortunately, the standard approach of carrying out decompositions on both the energy and pressure by using the same sequence of cutting functions $v_{k}=\left\{|u|-R\left(1-\frac{1}{2^{k}}\right)\right\}_{+}$is not powerful enough to ensure such a result as promised by proposition 2.1. So, in proving proposition 2.1, we will carry out the decomposition of the pressure $P$ by introducing another sequence of cutting functions $w_{k}=\left\{|u|-R^{\beta}\left(1-\frac{1}{2^{k}}\right)\right\}_{+}$, for $k \geqslant 1$, where $\beta>\frac{3}{2}$ should be some suitable index sufficiently close to $\frac{3}{2}$ (for more detail, see inequalities (4.3), (4.4), and (4.5) ). We remark that the inequality $\left\|\chi_{\left\{w_{k}>0\right\}}\right\|_{L^{q}\left(Q_{k-1}\right)} \leqslant \frac{2^{\frac{10 k}{3 q}}}{R^{\beta \frac{10}{3 q}}} C_{q} U_{k-1}^{\frac{5}{3 q}}$, for $q \geqslant 1$ provides us with the term $\frac{1}{R^{\frac{10 \beta}{3 q}}}$ which decays to 0 in a way much faster than $\frac{1}{R^{\frac{10}{3 q}}}$ as $R \rightarrow \infty$, and this is the reason why we use the cutting functions $w_{k}$ instead of $v_{k}$ in carrying out the decomposition of the pressure $P$.

Let us first show that Proposition 2.1 provides the result of Proposition 1.2. First, we show that the sequence $\left\{U_{k}\right\}_{k} \geqslant 1$ converges to 0 , when $k$ goes to infinity. We can use for instance the following easy lemma (see [19]):

Lemma 2.2. For any given constants $B, \beta>1$, there exists some constant $C_{0}^{*}$ such that for any sequence $\left\{a_{k}\right\}_{k \geqslant 1}$ satisfying $0<a_{1} \leq C_{0}^{*}$ and $a_{k} \leqslant B^{k} a_{k-1}^{\beta}$, for any $k \geqslant 1$, we have $\lim _{k \rightarrow \infty} a_{k}=0$.

With the assistance of lemma 2.2, we will derive the conclusion of proposition 1.2 from proposition 2.1 in the following way. Let $u$ be a suitable weak solution which satisfies the hypothesis of proposition 1.2. Then, according to inequality (2.1), which is the conclusion of proposition 2.1, we know that if the number $p$ with $1<p<\frac{5}{4}$ is chosen to be sufficiently close to 1 , and if the number $\beta>\frac{6-3 p}{10-8 p}$ is chosen to be sufficiently close to $\frac{3}{2}$, it follows that the sequence $\left\{U_{k}\right\}_{k=1}^{\infty}$ will satisfies the following inequality

$$
U_{k} \leqslant \frac{D}{R^{\Phi(p, \beta, \gamma)}} 2^{\frac{10 k}{3}}\left\{U_{k-1}^{\frac{5-p}{3 p}}+U_{k-1}^{\frac{5}{3 p}}+U_{k-1}^{\frac{5}{3}}\right\},
$$

in which the constants $D$ and $\Phi(p, \beta, \gamma)$ are given by

- $D=C_{p, \beta}\|u\|_{L^{\infty}\left(0,1 ; L^{2}\left(\mathbb{R}^{3}\right)\right)}^{2\left(1-\frac{1}{p}\right)}+(1+A)\left(1+\frac{1}{\alpha}\right)\left(1+K^{1-\frac{1}{p}}\right)\left(1+\|u\|_{L^{\infty}\left(0,1 ; L^{2}\left(\mathbb{R}^{3}\right)\right)}\right)$

- $\Phi(p, \beta, \gamma)=\min \left\{\beta\left(\frac{10-8 p}{3 p}\right)-\frac{2-p}{p},\left(\frac{10}{3}-2 p \beta+1-\gamma-p\right) \frac{1}{p},\left(\frac{10}{3}-2 \beta-\gamma\right)\right\}$. 
Notice that the constant $D$ depends on the choice of the suitable weak solution $u$ but independent of $R$, and $\Phi(p, \beta, \gamma)$ is some positive index which depends only on $p, \beta$, and $\gamma$.

Now, let us apply Lemma 2.2 to deduce that there is some constant $C_{0}^{*}$, such that for any sequence $\left\{a_{k}\right\}_{k=1}^{\infty}$ satisfying $0<a_{1} \leqslant C_{0}^{*}$ and $a_{k} \leqslant 2^{\frac{10 k}{3}} a_{k-1}^{\frac{5-p}{3 p}}$ for all $k \geqslant 1$, we have $\lim _{k \rightarrow \infty} a_{k}=0$. We then choose $R_{0}>1$ to be sufficiently large, so that we have $\frac{3 D}{R_{0}^{\Phi(p, \beta, \gamma)}}<1$, and that $U_{1} \leqslant \min \left\{\frac{1}{4}, C_{0}^{*}\right\}$. Then, notice that $d_{k}$ and $d_{1}$ are related by the following inequality

$$
\begin{aligned}
d_{k}^{2} & =\frac{R_{0}\left(1-\frac{1}{2^{k}}\right)}{\frac{R_{0}}{2}} \frac{\frac{R_{0}}{2}}{|u|} \chi_{\left\{|u|>R_{0}\left(1-\frac{1}{2^{k}}\right)\right\}}|\nabla| u||^{2}+\frac{v_{k}}{|u|}|\nabla u|^{2} \\
& \leqslant 2\left\{\left.\frac{\frac{R_{0}}{2}}{|u|} \chi_{\left\{|u|>\frac{R_{0}}{2}\right\}}|\nabla| u\right|^{2}+\frac{\left[|u|-\frac{R_{0}}{2}\right]+}{|u|}|\nabla u|^{2}\right\} \\
& \leqslant 2 d_{1}^{2}
\end{aligned}
$$

which, together with the definition of $U_{k}$ and the condition $U_{1} \leqslant \min \left\{\frac{1}{4}, C_{0}^{*}\right\}$, will imply that

$$
U_{k} \leqslant 2 U_{1} \leqslant \frac{1}{2}<1, \forall k \geqslant 1
$$

Since $\frac{5-p}{3 p}<\frac{5}{3 p}<\frac{5}{3}$ is valid for $1<p<\frac{5}{4}$, it follows from (2.3) and the condition $\frac{3 D}{R_{0}^{\Phi(p, \beta, \gamma)}}<1$ that the following estimation is valid for all $k \geqslant 2$

$$
U_{k} \leqslant \frac{D}{R_{0}^{\Phi(p, \beta, \gamma)}} 2^{\frac{10 k}{3}}\left\{U_{k-1}^{\frac{5-p}{3 p}}+U_{k-1}^{\frac{5}{3 p}}+U_{k-1}^{\frac{5}{3}}\right\} \leqslant \frac{3 D}{R_{0}^{\Phi(p, \beta, \gamma)}} 2^{\frac{10 k}{3}} U_{k-1}^{\frac{5-p}{3 p}} \leqslant 2^{\frac{10 k}{3}} U_{k-1}^{\frac{5-p}{3 p}} .
$$

With this choice of $R_{0}$, we see that the sequence $\left\{U_{k}\right\}_{k=1}^{\infty}$ will satisfy the conditions that $U_{1} \leqslant C_{0}^{*}$ and $U_{k} \leqslant 2^{\frac{10 k}{3}} U_{k-1}^{\frac{5-p}{3 p}}$, for all $k \geqslant 2$. Hence it follows that $\lim _{k \rightarrow \infty} U_{k}=0$. However, because for almost every $t \in\left[\frac{3}{4}, 1\right]$, we have

$$
\int_{\mathbb{R}^{3}}\left|u(t, x)-R_{0}\right|^{2} d x \leqslant 2 \lim _{k \rightarrow \infty} U_{k}=0 .
$$

It follows at once that $|u| \leqslant R_{0}$, almost everywhere over $\left[\frac{3}{4}, 1\right] \times \mathbb{R}^{3}$. This indicates that $u$ is essentially bounded over $\left[\frac{3}{4}, 1\right] \times \mathbb{R}^{3}$. Hence, we see that the conclusion of proposition 1.2 follows provided that proposition 2.1 is indeed valid.

For this reason, the main task of this paper is to give a detailed proof of proposition 2.1, which is what we will achieve in the following sections. More precisely, after we have given some preliminaries in section 3, we will actually carry out the proof of proposition 2.1 in section 4. Moreover, the proof of proposition 2.1 as presented in section 4 will be splitted into five successive steps. In step one, we will derive the inequality of the level set energy which gives an estimate of $U_{k}$ with respect to the pressure term $\int_{T_{k-1}}^{1}\left|\int_{\mathbb{R}^{3}} \frac{v_{k}}{|u|} u \nabla P d x\right| d s$. In step two, we will decompose the pressure $P$ into $P=P_{k 1}+P_{k 2}+P_{k 3}$ by using the cutting functions $w_{k}=\left\{|u|-R^{\beta}\left(1-\frac{1}{2^{k}}\right)\right\}_{+}$, with $\beta>\frac{3}{2}$ to be some sutiable index sufficiently close to $\frac{3}{2}$ (for more detail see equations 
(4.3), (4.4), and (4.5)). Here, we remark that $P_{k 2}$ and $P_{k 3}$ represent the effect of large velocity values $|u| \chi_{\left\{|u|>R^{\beta}\left(1-\frac{1}{2^{k}}\right)\right\}}$ on the pressure, while $P_{k 1}$ represents the effect of those velocity values smaller than $R^{\beta}\left(1-\frac{1}{2^{k}}\right)$ on the pressure. Step three is dedicated to the control of the two pressure terms involving big velocity values. Thanks to the introduction of the cutting functions $w_{k}=\left\{|u|-R^{\beta}\left(1-\frac{1}{2^{k}}\right)\right\}_{+}$in the decomposition of the pressure, the control on these two terms can then be performed successfully. In step four and step five, we will control the pressure term $\int_{T_{k-1}}^{1}\left|\int_{\mathbb{R}^{3}} \nabla\left(\frac{v_{k}}{|u|}\right) u P_{k 1} d x\right| d s$ which depends on those velocity values smaller than $R^{\beta}\left(1-\frac{1}{2^{k}}\right)$. In step four, we will show that such a pressure term depending on those velocity values smaller than $R^{\beta}\left(1-\frac{1}{2^{k}}\right)$ can be controlled by a weighted $|F| \log ^{+}|F|$ norm of $\operatorname{div}\left(\frac{u}{u}\right)$. We will finally show in step five that, in some specific way, we can eventually control the pressure term $\int_{T_{k-1}}^{1}\left|\int_{\mathbb{R}^{3}} \nabla\left(\frac{v_{k}}{|u|}\right) u P_{k 1} d x\right| d s$ successfully by employing the hypothesis $\frac{|u \cdot \nabla F|}{|u|^{\gamma}} \leqslant A|F|$ of proposition 2.1 .

\section{Preliminaries for the proof of proposition 2.1 .}

Lemma 3.1. There exists some constant $C>0$, such that for any $k \geqslant$ 1 , and any $f \in L^{\infty}\left(T_{k}, 1 ; L^{2}\left(\mathbb{R}^{3}\right)\right)$ with $\nabla f \in L^{2}\left(Q_{k}\right)$, we have $\|f\|_{L^{\frac{10}{3}}\left(Q_{k}\right)} \leqslant$ $C\|f\|_{L^{\infty}\left(T_{k}, 1 ; L^{2}\left(\mathbb{R}^{3}\right)\right)}^{\frac{2}{5}}\|\nabla f\|_{L^{2}\left(Q_{k}\right)}^{\frac{3}{5}}$.

Proof. By Sobolev-embedding Theorem, there is a constant $C$, depending only on the dimension of $\mathbb{R}^{3}$, such that

$$
\left(\int_{\mathbb{R}^{3}}|f(t, x)|^{6} d x\right)^{\frac{1}{6}} \leqslant C\left(\int_{\mathbb{R}^{3}}|\nabla f(t, x)|^{2} d x\right)^{\frac{1}{2}} .
$$

for any $t \in\left[T_{k}, 1\right]$, where $k \geqslant 1$, and $f$ is some function which verifies $f \in$ $L^{\infty}\left(T_{k}, 1 ; L^{2}\left(\mathbb{R}^{3}\right)\right)$, and $\nabla f \in L^{2}\left(Q_{k}\right)$. By taking power 2 on both sides of the above inequality and then taking integration along the variable $t \in\left[T_{k}, 1\right]$, we yield

$$
\int_{T_{k}}^{1}\left(\int_{\mathbb{R}^{3}}|f|^{6} d x\right)^{\frac{1}{3}} d t \leqslant C^{2} \int_{T_{k}}^{1} \int_{\mathbb{R}^{3}}|\nabla f|^{2} d x d t .
$$

On the other hand, by Holder's inequality, we have

$$
\begin{aligned}
\|f\|_{L^{\frac{10}{3}}\left(Q_{k}\right)}^{\frac{10}{3}} & =\int_{T_{k}}^{1} \int_{\mathbb{R}^{3}}|f|^{2}|f|^{\frac{4}{3}} d x d t \\
& \leqslant \int_{T_{k}}^{1}\left(\int_{\mathbb{R}^{3}}|f|^{6} d x\right)^{\frac{1}{3}}\left(\int_{\mathbb{R}^{3}}|f|^{2} d x\right)^{\frac{2}{3}} d t \\
& \leqslant\|f\|_{L^{\infty}\left(T_{k}, 1 ; L^{2}\left(\mathbb{R}^{3}\right)\right)}^{3}\|f\|_{L^{2}\left(T_{k}, 1 ; L^{6}\left(\mathbb{R}^{3}\right)\right)}^{2}
\end{aligned}
$$

By taking the advantage that $\|f\|_{L^{2}\left(T_{k}, 1 ; L^{6}\left(\mathbb{R}^{3}\right)\right)} \leqslant C\|\nabla f\|_{L^{2}\left(Q_{k}\right)}$, we yield

$$
\|f\|_{L^{\frac{10}{3}}\left(Q_{k}\right)}^{\frac{10}{3}} \leqslant C^{2}\|f\|_{L^{\infty}\left(T_{k}, 1 ; L^{2}\left(\mathbb{R}^{3}\right)\right)}^{\frac{4}{3}}\|\nabla f\|_{L^{2}\left(Q_{k}\right)}^{2} .
$$

Hence, we have 


$$
\|f\|_{L^{\frac{10}{3}}\left(Q_{k}\right)} \leqslant C\|f\|_{L^{\infty}\left(T_{k}, 1 ; L^{2}\left(\mathbb{R}^{3}\right)\right)}^{\frac{2}{5}}\|\nabla f\|_{L^{2}\left(Q_{k}\right)}^{\frac{3}{5}} .
$$

so, we are done.

Lemma 3.2. For any $1<q<\infty$, we have $\left\|\chi_{\left\{v_{k}>0\right\}}\right\|_{L^{q}\left(Q_{k-1}\right)} \leqslant \frac{2^{\frac{10 k}{3 q}}}{R^{\frac{10}{3 q}}} C^{\frac{1}{q}} U_{k-1}^{\frac{5}{3 q}}$. we have

Proof. First, we have to notice that $\left\{v_{k}>0\right\}$ is a subset of $\left\{v_{k-1}>\frac{R}{2^{k}}\right\}$, hence

$$
\int_{Q_{k-1}} \chi_{\left\{v_{k}>0\right\}} \leqslant \int_{Q_{k-1}} \chi_{\left\{v_{k-1}>\frac{R}{2^{k}}\right\}} \leqslant \frac{2^{\frac{10 k}{3}}}{R^{\frac{10}{3}}} \int_{Q_{k-1}}\left|v_{k-1}\right|^{\frac{10}{3}} .
$$

By our previous lemma, we have

$$
\begin{aligned}
\left\|v_{k-1}\right\|_{L^{\frac{10}{3}}}^{\frac{10}{3}}\left(Q_{k-1}\right) & \leqslant C^{2}\left\|v_{k-1}\right\|_{L^{\infty}\left(T_{k-1}, 1 ; L^{2}\left(\mathbb{R}^{3}\right)\right)}^{\frac{4}{3}}\left\|\nabla v_{k-1}\right\|_{L^{2}\left(Q_{k-1}\right)}^{2} \\
& \leqslant C^{2}\left(U_{k-1}^{\frac{1}{2}}\right)^{\frac{4}{3}}\left\|d_{k-1}\right\|_{L^{2}\left(Q_{k-1}\right)}^{2} \\
& \leqslant C^{2} U_{k-1}^{\frac{2}{3}} U_{k-1} \\
& =C^{2} U_{k-1}^{\frac{5}{3}} .
\end{aligned}
$$

So, it follows that $\int_{Q_{k-1}} \chi_{\left\{v_{k}>0\right\}} \leqslant \frac{2^{\frac{10 k}{3}}}{R^{\frac{10}{3}}} C^{2} U_{k-1}^{\frac{5}{3}}$, and hence we have

$$
\left\|\chi_{\left\{v_{k}>0\right\}}\right\|_{L^{q}\left(Q_{k-1}\right)} \leqslant \frac{2^{\frac{10 k}{3 q}}}{R^{\frac{10}{3 q}}} C^{\frac{1}{q}} U_{k-1}^{\frac{5}{3 q}},
$$

in which $C$ is some universal constant. So, we are done. $\square$

Just as we have said before, we will need to decompose the pressure by employing the sequence of cutting functions $w_{k}=\left\{|u|-R^{\beta}\left(1-\frac{1}{2^{k}}\right)\right\}_{+}$, for $k \geqslant 1$. We also said that we prefer to do this because the cutting functions $w_{k}$ satisfy the following inequality which can be justified in the same way as Lemma 3.2.

Lemma 3.3. For every $q \geqslant 1$, we have $\left\|\chi_{\left\{w_{k}>0\right\}}\right\|_{L^{q}\left(Q_{k-1}\right)} \leqslant \frac{1}{R^{\frac{10 \beta}{3 q}}} 2^{\frac{10 k}{3 q}} C_{q} U_{k-1}^{\frac{5}{3 q}}$, for all $k \geqslant 1$, in which $C_{q}$ is some constant depending only on $q$.

Indeed, in dealing with the pressure terms, we will invoke the Lemma 3.3 without explicit mention.

In the proof of Lemma 3.2, we have used the fact that $\left|\nabla v_{k}\right| \leqslant d_{k}$, whose justification will be given immediately in the following paragraph.

Before we leave this section, we also want to list out some inequalities which will often be used in the proof of proposition 2.1 as follow:

- $\left|\left(1-\frac{v_{k}}{|u|}\right) u\right| \leqslant R\left(1-\frac{1}{2^{k}}\right)$.

- $\frac{v_{k}}{|u|}|\nabla u| \leqslant d_{k}$.

- $\chi_{\left\{v_{k} \geqslant 0\right\}}|\nabla| u|| \leqslant d_{k}$.

- $\left|\nabla v_{k}\right| \leqslant d_{k}$. 
- $\left|\nabla\left(\frac{v_{k}}{|u|} u\right)\right| \leqslant 3 d_{k}$.

Now, we first want to justify the validity of $\left|\left(1-\frac{v_{k}}{\mid u}\right) u\right| \leqslant R\left(1-\frac{1}{2^{k}}\right)$. In the case in which the point $(t, x)$ satisfies $|u(t, x)|<R\left(1-\frac{1}{2^{k}}\right)$, we have $v_{k}(t, x)=0$, and hence it follows that

$$
\left|\left\{1-\frac{v_{k}(t, x)}{|u(t, x)|}\right\} u(t, x)\right|=|u(t, x)|<R\left(1-\frac{1}{2^{k}}\right) .
$$

In the case in which $(t, x)$ satisfies $|u(t, x)| \geqslant R\left(1-\frac{1}{2^{k}}\right)$, we have $v_{k}(t, x)=|u(t, x)|-$ $R\left(1-\frac{1}{2^{k}}\right)$, and hence it follows that

$$
\left|\left\{1-\frac{v_{k}}{|u|}\right\} u(t, x)\right|=\left|1-\frac{|u|-R\left(1-\frac{1}{2^{k}}\right)}{|u|}\right||u|=R\left(1-\frac{1}{2^{k}}\right) .
$$

So, no matter in which case, we always have the conclusion that $\left|\left(1-\frac{v_{k}}{|u|}\right) u\right| \leqslant$ $R\left(1-\frac{1}{2^{k}}\right)$.

Next, according to the definition of $d_{k}^{2}$, we can carry out the following estimation

$$
d_{k}^{2} \geqslant \frac{v_{k}}{|u|}|\nabla u|^{2} \geqslant\left\{\frac{v_{k}}{|u|}|\nabla u|\right\}^{2} .
$$

Hence, by taking square root, it follows at once that $d_{k} \geqslant \frac{v_{k}}{|u|}|\nabla u|$.

We now turn our attention to the inequality $\chi_{\left\{|u|>R\left(1-\frac{1}{2^{k}}\right)\right\}}|\nabla| u|| \leqslant d_{k}$. To justify it, we recall that $|\nabla u| \geqslant|\nabla| u||$. Hence, it follows from the definition of $d_{k}^{2}$ that

$$
d_{k}^{2} \geqslant \frac{R\left(1-\frac{1}{2^{k}}\right)}{|u|} \chi_{\left\{|u|>R\left(1-\frac{1}{2^{k}}\right)\right\}}|\nabla| u||^{2}+\left\{1-\frac{R\left(1-\frac{1}{2^{k}}\right)}{|u|}\right\} \chi_{\left\{|u|>R\left(1-\frac{1}{2^{k}}\right)\right\}}|\nabla| u||^{2} .
$$

So, by simplifying the right-hand side of the above inequality, we can deduce that $d_{k}^{2} \geqslant \chi_{\left\{|u|>R\left(1-\frac{1}{2^{k}}\right)\right\}}|\nabla| u||^{2}$. Hence, we have $d_{k} \geqslant \chi_{\left\{|u|>R\left(1-\frac{1}{2^{k}}\right)\right\}}|\nabla| u||$. In addition, since it is obvious to see that $\nabla v_{k}=\chi_{\left\{|u|>R\left(1-\frac{1}{2^{k}}\right)\right\}} \nabla|u|$, we also have the result that $\left|\nabla v_{k}\right| \leqslant d_{k}$

Finally, we want to justify the inequality that $\left|\nabla\left(\frac{v_{k}}{|u|} u\right)\right| \leqslant 3 d_{k}$. So, we notice that, by applying the product rule, we have

$$
\nabla\left(\frac{v_{k}}{|u|} u\right)=\nabla\left(v_{k}\right) \frac{u}{|u|}+\frac{v_{k}}{|u|} \nabla u-\frac{v_{k}}{|u|^{2}} u \nabla|u| .
$$

However, since $\frac{v_{k}}{|u|}|\nabla u| \leqslant d_{k}$, and $\left|\frac{v_{k}}{|u|^{2}} u \nabla\right| u|| \leqslant \chi_{\left\{|u|>R\left(1-\frac{1}{2^{k}}\right)\right\}}|\nabla| u|| \leqslant d_{k}$, it follows at once from the above expression that $\left|\nabla\left(\frac{v_{k}}{|u|} u\right)\right| \leqslant 3 d_{k}$.

\section{Proof of proposition 2.1.}

Step one. To begin the argument, we recall that, according to Lemma 5 in [19], the truncations $v_{k}=\left\{|u|-R\left(1-\frac{1}{2^{k}}\right)\right\}$ of a given suitable weak solution $u:[0,1] \times \mathbb{R}^{3} \rightarrow$ $\mathbb{R}^{3}$ satisfy the following inequality in the sense of distribution.

$$
\partial_{t}\left(\frac{v_{k}^{2}}{2}\right)+d_{k}^{2}-\triangle\left(\frac{v_{k}^{2}}{2}\right)+\operatorname{div}\left(\frac{v_{k}^{2}}{2} u\right)+\frac{v_{k}}{|u|} u \nabla P \leqslant 0 .
$$


As mentioned in Remark 2 of [19], inequality (4.1) can not be derived from the notion of Leray-Hopf weak solutions alone, and the validity of inequality (4.1) is based on the local energy inequality (1.2) which characterizes the notion of suitable weak solutions.

Next, let us consider the variables $\sigma, t$ verifying $T_{k-1} \leqslant \sigma \leqslant T_{k} \leqslant t \leqslant 1$. Then, we have

- $\int_{\sigma}^{t} \int_{\mathbb{R}^{3}} \partial_{t}\left(\frac{v_{k}^{2}}{2}\right) d x d s=\int_{\mathbb{R}^{3}} \frac{v_{k}^{2}(t, x)}{2} d x-\int_{\mathbb{R}^{3}} \frac{v_{k}^{2}(\sigma, x)}{2} d x$.

- $\int_{\sigma}^{t} \int_{\mathbb{R}^{3}} \triangle\left(\frac{v_{k}^{2}}{2}\right) d x d s=0$.

- $\int_{\sigma}^{t} \int_{\mathbb{R}^{3}} \operatorname{div}\left(\frac{v_{k}^{2}}{2} u\right) d x d s=0$.

So, it is straightforward to see that

$$
\int_{\mathbb{R}^{3}} \frac{v_{k}^{2}(t, x)}{2} d x+\int_{\sigma}^{t} \int_{\mathbb{R}^{3}} d_{k}^{2} d x d s \leqslant \int_{\mathbb{R}^{3}} \frac{v_{k}^{2}(\sigma, x)}{2} d x+\int_{\sigma}^{t}\left|\int_{\mathbb{R}^{3}} \frac{v_{k}}{|u|} u \nabla P d x\right| d s,
$$

for any $\sigma, t$ satisfying $T_{k-1} \leqslant \sigma \leqslant T_{k} \leqslant t \leqslant 1$. By taking the average over the variable $\sigma$, we yield

$$
\begin{aligned}
& \int_{\mathbb{R}^{3}} \frac{v_{k}^{2}(t, x)}{2} d x+\int_{T_{k}}^{t} \int_{\mathbb{R}^{3}} d_{k}^{2} d x d s \\
\leqslant & \frac{4^{k+1}}{6} \int_{T_{k-1}}^{T_{k}} \int_{\mathbb{R}^{3}} v_{k}^{2}(s, x) d x d s+\int_{T_{k-1}}^{t}\left|\int_{\mathbb{R}^{3}} \frac{v_{k}}{|u|} u \nabla P d x\right| d s .
\end{aligned}
$$

By taking the sup over $t \in\left[T_{k}, 1\right]$. the above inequality will give the following

$$
U_{k} \leqslant \frac{4^{k+1}}{6} \int_{Q_{k-1}} v_{k}^{2}+\int_{T_{k-1}}^{1}\left|\int_{\mathbb{R}^{3}} \frac{v_{k}}{|u|} u \nabla P d x\right| d s .
$$

But, from Lemma 3.2 and Holder's inequality, we have

$$
\begin{aligned}
\int_{Q_{k-1}} v_{k}^{2} & =\int_{Q_{k-1}} v_{k}^{2} \chi_{\left\{v_{k}>0\right\}} \\
& \leqslant\left(\int_{Q_{k-1}} v_{k}^{\frac{10}{3}}\right)^{\frac{3}{5}}\left\|\chi_{\left\{v_{k}>0\right\}}\right\|_{L^{\frac{5}{2}}\left(Q_{k-1}\right)} \\
& \leqslant\left\|v_{k}\right\|_{L^{\frac{10}{3}}}^{2}\left(Q_{k-1}\right) \frac{2^{\frac{4 k}{3}}}{R^{\frac{4}{3}}} C^{\frac{2}{5}} U_{k-1}^{\frac{2}{3}} \\
& \leqslant\left\|v_{k-1}\right\|_{L^{\frac{10}{3}}}^{2}\left(Q_{k-1}\right) \frac{2^{\frac{4 k}{3}}}{R^{\frac{4}{3}}} C^{\frac{2}{5}} U_{k-1}^{\frac{2}{3}} \\
& \leqslant C U_{k-1}^{\frac{5}{3}} \frac{2^{\frac{4 k}{3}}}{R^{\frac{4}{3}}} .
\end{aligned}
$$

As a result, we have the following conclusion

$$
U_{k} \leqslant \frac{2^{\frac{10 k}{3}}}{R^{\frac{4}{3}}} C U_{k-1}^{\frac{5}{3}}+\int_{T_{k-1}}^{1}\left|\int_{\mathbb{R}^{3}} \frac{v_{k}}{|u|} u \nabla p d x\right| d s
$$


Step two. Now, in order to estimate the term $\int_{T_{k-1}}^{1}\left|\int_{\mathbb{R}^{3}} \frac{v_{k}}{|u|} u \nabla P d x\right| d s$, we would like to carry out the following computation

$$
\begin{aligned}
-\triangle P & =\sum \partial_{i} \partial_{j}\left(u_{i} u_{j}\right) \\
& =\sum \partial_{i} \partial_{j}\left\{\left(1-\frac{w_{k}}{|u|}\right) u_{i}\left(1-\frac{w_{k}}{|u|}\right) u_{j}\right\}+2 \sum \partial_{i} \partial_{j}\left\{\left(1-\frac{w_{k}}{|u|}\right) u_{i} \frac{w_{k}}{|u|} u_{j}\right\} \\
& +\sum \partial_{i} \partial_{j}\left\{\frac{w_{k}}{|u|} u_{i} \frac{w_{k}}{|u|} u_{j}\right\},
\end{aligned}
$$

in which $w_{k}$ is given by $w_{k}=\left\{|u|-R^{\beta}\left(1-\frac{1}{2^{k}}\right)\right\}_{+}$, and $\beta$ is simply the arbritary index involved in proposition 2.1. This motivates us to decompose $P$ as $P=P_{k 1}+P_{k 2}+P_{k 3}$, in which

$$
\begin{gathered}
-\triangle P_{k 1}=\sum \partial_{i} \partial_{j}\left\{\left(1-\frac{w_{k}}{|u|}\right) u_{i}\left(1-\frac{w_{k}}{|u|}\right) u_{j}\right\} \\
-\triangle P_{k 2}=\sum \partial_{i} \partial_{j}\left\{2\left(1-\frac{w_{k}}{|u|}\right) u_{i} \frac{w_{k}}{|u|} u_{j}\right\} \\
-\triangle P_{k 3}=\sum \partial_{i} \partial_{j}\left\{\frac{w_{k}}{|u|} u_{i} \frac{w_{k}}{|u|} u_{j}\right\} .
\end{gathered}
$$

Here, we have to remind ourself that the cutting functions which are used in the decomposition of the pressure are indeed $w_{k}=\left\{|u|-R^{\beta}\left(1-\frac{1}{2^{k}}\right)\right\}_{+}$, for all $k \geqslant 0$, in which $\beta$ is some suitable index strictly greater than $\frac{3}{2}$. With respect to the cutting functions $w_{k}$, we need to define the respective $D_{k}$ as follow:

$$
D_{k}^{2}=\frac{R^{\beta}\left(1-\frac{1}{2^{k}}\right)}{|u|} \chi_{\left\{w_{k}>0\right\}}|\nabla| u||^{2}+\frac{w_{k}}{|u|}|\nabla u|^{2} .
$$

Then, just like what happens to the cutting functions $v_{k}$, we have the following assertions about the cutting functions $w_{k}$, which are easily verified.

- $\left|\nabla w_{k}\right| \leqslant D_{k}$, for all $k \geqslant 0$.

- $\left|\nabla\left(\frac{w_{k}}{|u|} u_{i}\right)\right| \leqslant 3 D_{k}$, for all $k \geqslant 0$, and $1 \leqslant i \leqslant 3$.

- $\left|\nabla\left(\frac{w_{k}}{|u|}\right) u_{i}\right| \leqslant 2 D_{k}$, for any $k \geqslant 0$, and $1 \leqslant i \leqslant 3$.

Besides these, we also need the following lemma which links $D_{k}$ to $d_{k}$.

LEMma 4.1. There is some sufficiently large $R_{0}>1$, such that whenever $R>R_{0}$ and $k \geqslant 1$, we have $D_{k} \leqslant 5^{\frac{1}{2}} d_{k}$.

Proof. Since $\frac{R^{\beta}-R}{R^{\beta}}$ trends to the limiting value 1 , as $R$ trends to $\infty$. So, there is some sufficiently large $R_{0}>1$ for which $\left(R^{\beta}-R\right)>\frac{R^{\beta}}{2}$, for all $R>R_{0}$. Now, notice that $\left\{w_{k}>0\right\}$ is a subset of $\left\{v_{k}>\left(R^{\beta}-R\right)\left(1-\frac{1}{2^{k}}\right)\right\}$, for all $k \geqslant 0$. Hence, it follows that $\left\{w_{k}>0\right\}$ is a subset of $\left\{v_{k}>\frac{R^{\beta}}{4}\right\}$, for all $k \geqslant 1$ and $R>R_{0}$. As a result, we can carry out the following computation 


$$
\begin{aligned}
D_{k}^{2} & =\frac{R^{\beta}\left(1-\frac{1}{2^{k}}\right)}{|u|} \chi_{\left\{w_{k}>0\right\}}|\nabla| u||^{2}+\frac{w_{k}}{|u|}|\nabla u|^{2} \\
& \leqslant\left.\frac{R^{\beta}}{|u|} \chi_{\left\{w_{k}>0\right\}}|\nabla| u\right|^{2}+\frac{v_{k}}{|u|}|\nabla u|^{2} \\
& \leqslant\left.\frac{4 v_{k}}{|u|} \chi_{\left\{w_{k}>0\right\}}|\nabla| u\right|^{2}+\frac{v_{k}}{|u|}|\nabla u|^{2} \\
& \leqslant \frac{5 v_{k}}{|u|}|\nabla u|^{2} \leqslant 5 d_{k}^{2},
\end{aligned}
$$

for any $k \geqslant 1$, and $R>R_{0}$. Hence, we have $D_{k} \leqslant 5^{\frac{1}{2}} d_{k}$, for all $k \geqslant 1$, and all $R>R_{0}$. So, we are done. $\square$

Now, let us recall that we have already used the cutting functions $w_{k}$ to obtain the decomposition $P=P_{k 1}+P_{k 2}+P_{k 3}$, in which $P_{k 1}, P_{k 2}$, and $P_{k 3}$ are described in equations (4.3), (4.4), and (4.5) respectively.

Due to the incompressible condition $\operatorname{div}(u)=0$, we have the following two identities

- $\int_{\mathbb{R}^{3}} \frac{v_{k}}{|u|} u \nabla P_{k 2} d x=\int_{\mathbb{R}^{3}}\left(\frac{v_{k}}{|u|}-1\right) u \nabla P_{k 2} d x$.

- $\int_{\mathbb{R}^{3}} \frac{v_{k}}{|u|} u \nabla P_{k 3} d x=\int_{\mathbb{R}^{3}}\left(\frac{v_{k}}{|u|}-1\right) u \nabla P_{k 3} d x$.

Hence, it follows that

$$
\begin{aligned}
\int_{T_{k-1}}^{1}\left|\int_{\mathbb{R}^{3}} \frac{v_{k}}{|u|} u \nabla P d x\right| d t & \leqslant \int_{T_{k-1}}^{1}\left|\int_{\mathbb{R}^{3}} \nabla\left(\frac{v_{k}}{|u|}\right) u P_{k 1} d x\right| d t+\int_{Q_{k-1}}\left(1-\frac{v_{k}}{|u|}\right)|u|\left|\nabla P_{k 2}\right| \\
& +\int_{Q_{k-1}}\left(1-\frac{v_{k}}{|u|}\right)|u|\left|\nabla P_{k 3}\right| .
\end{aligned}
$$

Step 3. We are now ready to deal with the term $\int_{Q_{k-1}}\left(1-\frac{v_{k}}{|u|}\right)|u|\left|\nabla P_{k 2}\right|$. For this purpose, let $p$ be such that $1<p<\frac{5}{4}$, and let $q=\frac{p}{p-1}$, so that $2<q<\infty$. We remark that the purpose of the condition $1<p<\frac{5}{4}$ is to ensure that the quantity $\frac{2 p}{2-p}$ will satisfy the condition $2<\frac{2 p}{2-p}<\frac{10}{3}$, which is required in the forthcoming inequality estimation (4.8). Next, by applying Holder's inequality, we find that

$$
\begin{aligned}
\left\|\left(1-\frac{v_{k}}{|u|}\right) u\right\|_{L^{q}\left(\mathbb{R}^{3}\right)} & \leqslant\left\|\left(1-\frac{v_{k}}{|u|}\right) u\right\|_{L^{2}\left(\mathbb{R}^{3}\right)}^{\frac{2}{q}}\left\|\left(1-\frac{v_{k}}{|u|}\right) u\right\|_{L^{\infty}\left(\mathbb{R}^{3}\right)}^{1-\frac{2}{q}} \\
& \leqslant R^{1-\frac{2}{q}}\left\|\left(1-\frac{v_{k}}{|u|}\right) u\right\|_{L^{2}\left(\mathbb{R}^{3}\right)}^{\frac{2}{q}} \\
& \leqslant R^{\frac{2}{p}-1}\|u\|_{L^{\infty}\left(0,1 ; L^{2}\left(\mathbb{R}^{3}\right)\right)}^{2\left(1-\frac{1}{p}\right)}
\end{aligned}
$$

Hence, it follows from Holder's inequality that

$$
\int_{\mathbb{R}^{3}}\left(1-\frac{v_{k}}{|u|}\right)|u|\left|\nabla P_{k 2}\right| d x \leqslant R^{\frac{2}{p}-1}\|u\|_{L^{\infty}\left(0,1 ; L^{2}\left(\mathbb{R}^{3}\right)\right)}^{2\left(1-\frac{1}{2}\right)}\left\{\int_{\mathbb{R}^{3}}\left|\nabla P_{k 2}\right|^{p} d x\right\}^{\frac{1}{p}}
$$

Hence, we have 


$$
\int_{Q_{k-1}}\left(1-\frac{v_{k}}{|u|}\right)|u|\left|\nabla P_{k 2}\right| \leqslant R^{\frac{2}{p}-1}\|u\|_{L^{\infty}\left(0,1 ; L^{2}\left(\mathbb{R}^{3}\right)\right)}^{2\left(1-\frac{1}{p}\right)}\left\|\nabla P_{k 2}\right\|_{L^{p}\left(Q_{k-1}\right)} .
$$

But, we recognize that

$\nabla P_{k 2}=\sum R_{i} R_{j}\left\{2\left(1-\frac{w_{k}}{|u|}\right) u_{i} \nabla\left[\frac{w_{k}}{|u|} u_{j}\right]+2\left(1-\frac{w_{k}}{|u|}\right) u_{j}\left[\frac{w_{k}}{|u|} \nabla u_{i}\right]-2 \nabla\left[\frac{w_{k}}{|u|}\right] u_{i} \frac{w_{k}}{|u|} u_{j}\right\}$.

Moreover, it is straightforward to see that for any $1 \leqslant i, j \leqslant 3$, we have

- $\left|2\left(1-\frac{w_{k}}{|u|}\right) u_{i} \nabla\left[\frac{w_{k}}{|u|} u_{j}\right]+2\left(1-\frac{w_{k}}{|u|}\right) u_{j}\left[\frac{w_{k}}{|u|} \nabla u_{i}\right]\right| \leqslant 8 R^{\beta} D_{k}$.

- $\left|2 \nabla\left[\frac{w_{k}}{|u|}\right] u_{i} \frac{w_{k}}{|u|} u_{j}\right| \leqslant 8 w_{k} D_{k}$.

So, we can decompose $\nabla P_{k 2}$ as $\nabla P_{k 2}=G_{k 21}+G_{k 22}$, where $G_{k 21}$ and $G_{k 22}$ are given by

- $G_{k 21}=\sum R_{i} R_{j}\left\{2\left(1-\frac{w_{k}}{|u|}\right) u_{i} \nabla\left[\frac{w_{k}}{|u|} u_{j}\right]+2\left(1-\frac{w_{k}}{|u|}\right) u_{j}\left[\frac{w_{k}}{|u|} \nabla u_{i}\right]\right\}$.

- $G_{k 22}=-\sum R_{i} R_{j}\left\{2 \nabla\left[\frac{w_{k}}{|u|}\right] u_{i} \frac{w_{k}}{|u|} u_{j}\right\}$.

In order to use inequality (4.6), we need to estimate $\left\|G_{k 21}\right\|_{L^{p}\left(Q_{k-1}\right)}$ and $\left\|G_{k 22}\right\|_{L^{p}\left(Q_{k-1}\right)}$ respectively, for $p$ with $1<p<\frac{5}{4}$. Indeed, by applying the ZygmundCalderon Theorem, we can deduce that

- $\left\|G_{k 21}\right\|_{L^{p}\left(Q_{k-1}\right)} \leqslant C_{p} R^{\beta}\left\|D_{k}\right\|_{L^{p}\left(Q_{k-1}\right)}$,

- $\left\|G_{k 22}\right\|_{L^{p}\left(Q_{k-1}\right)} \leqslant C_{p}\left\|w_{k} D_{k}\right\|_{L^{p}\left(Q_{k-1}\right)}$,

where $C_{p}$ is some constant depending only on $p$. But it turns out that

$$
\begin{aligned}
\left\|D_{k}\right\|_{L^{p}\left(Q_{k-1}\right)}^{p} & =\int_{Q_{k-1}} D_{k}^{p} \chi_{\left\{w_{k}>0\right\}} \\
& \leqslant\left\{\int_{Q_{k-1}} D_{k}^{2}\right\}^{\frac{p}{2}}\left\|\chi_{\left\{w_{k}>0\right\}}\right\|_{L^{\frac{2}{2-p}}\left(Q_{k-1}\right)} \\
& \leqslant \frac{5^{\frac{p}{2}}}{R^{\frac{5}{3} \beta(2-p)}}\left\|d_{k}\right\|_{L^{2}\left(Q_{k-1}\right)}^{2^{\frac{5 k}{3}(2-p)}} C_{p} U_{k-1}^{\frac{5}{6}(2-p)} \\
& \leqslant \frac{1}{R^{\frac{5}{3} \beta(2-p)}} C_{p} U_{k-1}^{\frac{5-p}{3}} 2^{\frac{5(2-p) k}{3}} .
\end{aligned}
$$

That is, we have

$$
\left\|D_{k}\right\|_{L^{p}\left(Q_{k-1}\right)} \leqslant \frac{1}{R^{\frac{5}{3 p} \beta(2-p)}} C_{p} U_{k-1}^{\frac{5-p}{3 p}} 2^{\frac{5(2-p) k}{3 p}} .
$$

Hence, it follows that

$$
\left\|G_{k 21}\right\|_{L^{p}\left(Q_{k-1}\right)} \leqslant \frac{1}{R^{\beta\left(\frac{10-8 p}{3 p}\right)}} C_{p} U_{k-1}^{\frac{5-p}{3 p}} 2^{\frac{5(2-p) k}{3 p}} .
$$

On the other hand, we have

$$
\begin{aligned}
\left\|w_{k} D_{k}\right\|_{L^{p}\left(Q_{k-1}\right)}^{p} & =\int_{Q_{k-1}} w_{k}^{p} D_{k}^{p} \\
& \leqslant\left\{\int_{Q_{k-1}} w_{k}^{\frac{2 p}{2-p}}\right\}^{\frac{2-p}{2}}\left\{\int_{Q_{k-1}} D_{k}^{2}\right\}^{\frac{p}{2}} \\
& \leqslant C_{p}\left\{\int_{Q_{k-1}} w_{k}^{\frac{2 p}{2-p}}\right\}^{\frac{2-p}{2}} U_{k-1}^{\frac{p}{2}} .
\end{aligned}
$$


Now, let us recall that $1<p<\frac{5}{4}$, and put $r=\frac{2 p}{2-p}$. we then recognize that $2<r=\frac{2 p}{2-p}<\frac{10}{3}$, if $1<p<\frac{5}{4}$. So, we can have the following estimation

$$
\begin{aligned}
\int_{Q_{k-1}} w_{k}^{\frac{2 p}{2-p}} & =\int_{Q_{k-1}} w_{k}^{r} \chi_{\left\{w_{k}>0\right\}} \\
& \leqslant \int_{Q_{k-1}} w_{k}^{r} \chi_{\left\{w_{k-1}>\frac{R^{\beta}}{2^{k}}\right\}} \\
& \leqslant \frac{1}{R^{\beta\left(\frac{10}{3}-r\right)}} 2^{k\left(\frac{10}{3}-r\right)} \int_{Q_{k-1}} w_{k}^{\frac{10}{3}} \\
& \leqslant \frac{1}{R^{\beta \frac{20-16 p}{3(2-p)}}} 2^{\frac{k(20-16 p)}{3(2-p)}} U_{k-1}^{\frac{5}{3}} .
\end{aligned}
$$

Hence, it follows that

$$
\left\|G_{k 22}\right\|_{L^{p}\left(Q_{k-1}\right)} \leqslant\left\|w_{k} D_{k}\right\|_{L^{p}\left(Q_{k-1}\right)} \leqslant C_{p} \frac{2^{k \frac{10-8 p}{3 p}}}{R^{\beta \frac{10-8 p}{3 p}}} U_{k-1}^{\frac{5-p}{3 p}} .
$$

By combining inequalities (4.6), (4.7), (4.9), we deduce that

$$
\int_{Q_{k-1}}\left(1-\frac{v_{k}}{|u|}\right)|u|\left|\nabla P_{k 2}\right| \leqslant \frac{1}{R^{\beta \frac{10-8 p}{3 p}-\frac{2-p}{p}}} C_{p}\|u\|_{L^{\infty}\left(0,1 ; L^{2}\left(\mathbb{R}^{3}\right)\right)}^{2\left(1-\frac{1}{p}\right)} U_{k-1}^{\frac{5-p}{3 p}} 2^{\frac{10-5 p}{3 p} k}
$$

Notice that $\beta\left(\frac{10-8 p}{3 p}\right)-\left(\frac{2-p}{p}\right)>0$ if and only if $\beta>\frac{6-3 p}{10-8 p}$. Moreover, we know that the term $\frac{6-3 p}{10-8 p}$ is always positive, for $1<p<\frac{5}{4}$. In addition, we know that as $p$ trends to $1, \frac{6-3 p}{10-8 p}$ trends to $\frac{3}{2}$. This means that even though $\beta$ cannot be exactly $\frac{3}{2}$, $\beta>\frac{3}{2}$ can be adjusted to be as close to $\frac{3}{2}$ as we desire.

As for the term $\int_{Q_{k-1}}\left(1-\frac{v_{k}}{|u|}\right)|u|\left|\nabla P_{k 3}\right|$. We first notice that

$$
P_{k 3}=\sum R_{i} R_{j}\left\{\frac{w_{k}}{|u|} u_{i} \frac{w_{k}}{|u|} u_{j}\right\}
$$

So, we know that

$$
\nabla P_{k 3}=\sum R_{i} R_{j}\left\{\nabla\left[\frac{w_{k}}{|u|} u_{i}\right] \frac{w_{k}}{|u|} u_{j}+\frac{w_{k}}{|u|} u_{i} \nabla\left[\frac{w_{k}}{|u|} u_{j}\right]\right\}
$$

with

$$
\left|\nabla\left[\frac{w_{k}}{|u|} u_{i}\right] \frac{w_{k}}{|u|} u_{j}+\frac{w_{k}}{|u|} u_{i} \nabla\left[\frac{w_{k}}{|u|} u_{j}\right]\right| \leqslant 6 w_{k} D_{k}
$$

So, it follows again from the Risez's theorem that $\left\|\nabla P_{k 3}\right\|_{L^{p}\left(\mathbb{R}^{3}\right)} \leqslant$ $C_{p}\left\|w_{k} D_{k}\right\|_{L^{p}\left(\mathbb{R}^{3}\right)}$, in which $C_{p}$ is some constant depending only on $p$. So, we see that we can repeat the same type of estimation, just as what we have done to the term $\int_{Q_{k-1}}\left(1-\frac{v_{k}}{|u|}\right)|u|\left|\nabla P_{k 2}\right|$, to conclude that 


$$
\begin{aligned}
\int_{Q_{k-1}}\left(1-\frac{v_{k}}{|u|}\right)|u|\left|\nabla P_{k 3}\right| & \leqslant R^{\frac{2}{p}-1}\|u\|_{L^{\infty}\left(0,1 ; L^{2}\left(\mathbb{R}^{3}\right)\right)}^{2\left(1-\frac{1}{p}\right)}\left\|\nabla P_{k 3}\right\|_{L^{p}\left(Q_{k-1}\right)} \\
& \leqslant \frac{C_{p}\|u\|_{L^{\infty}\left(0,1 ; L^{2}\left(\mathbb{R}^{3}\right)\right)}^{2\left(1-\frac{1}{p}\right)}}{R^{\beta \frac{10-8 p}{3 p}-\frac{2-p}{p}}} U_{k-1}^{\frac{5-p}{3 p}} 2^{\frac{(10-5 p) k}{3 p}}
\end{aligned}
$$

Step four. Now, let us turn our attention to the term $\int_{T_{k-1}}^{1}\left|\int_{\mathbb{R}^{3}} \nabla\left(\frac{v_{k}}{|u|}\right) u P_{k 1} d x\right| d s$. Before we deal with the term written as above, let us recall that the weak solution $u$ that we are dealing with now is the one verifying the following condition

$$
\frac{|u \cdot \nabla F|}{|u|^{\gamma}} \leqslant A|F|
$$

where $F=-\frac{u \cdot \nabla|u|}{|u|^{2}}$, and $\gamma$ is some index with $0<\gamma<\frac{1}{3}$. We need to introduce the following classical theorem of harmonic analysis which is due to John and Nirenberg $[9]$.

THEOREM 4.2. Let $B$ be a ball with finite radius sitting in $\mathbb{R}^{3}$. Then, there exists some constants $\alpha$, and $K$, with $0<\alpha<\infty$, and $0<K<\infty$, depending only on the ball $B$ and $n$, such that for any given $f \in B M O\left(\mathbb{R}^{n}\right)$, we have $\int_{B} \exp \left(\alpha \frac{\left|f-f_{B}\right|}{\mid f \|_{B M O}}\right) \leqslant K$, where the symbol $f_{B}$ stands for the mean value of $f$ over $B$.

We now need to establish the following lemma by using the theorem quoted as above.

Lemma 4.3. Let $B$ be a ball with finite radius sitting in $\mathbb{R}^{3}$. There exists some finite positive constants $\alpha$ and $K$, depending only on $B$, such that for every $\mu \geqslant 0$, every $f \in B M O\left(\mathbb{R}^{3}\right)$ with $\int_{B} f d x=0$, and $p$ with $1<p<\infty$, we have $\int_{B} \mu|f| \leqslant$ $\frac{2 p}{\alpha(p-1)}\left\{1+K^{1-\frac{1}{p}}\right\}\|f\|_{B M O}\left\{\left(\int_{B} \mu\right)^{\frac{1}{p}}+\int_{B} \mu \log ^{+} \mu\right\}$.

Proof. For any given $\mu \geqslant 0$, and any $f \in B M O\left(\mathbb{R}^{3}\right)$ with $\int_{B} f d x=0$, we do the following splitting

$$
\int_{B} \mu|f|=\int_{B} \mu|f| \chi_{\left\{\mu \leqslant \exp \left(\frac{\alpha|f|}{2\|f\|_{B M O}}\right)\right\}}+\int_{B} \mu|f| \chi_{\left\{\mu>\exp \left(\frac{\alpha|f|}{2\|f\|_{B M O}}\right)\right\}} .
$$

Given $p$ be such that $1<p<\infty$, and let $q=\frac{p}{p-1}$ be the conjugate exponent of $p$. So, it follows from Holder's inequality that

$$
\begin{aligned}
& \int_{B} \mu|f| \chi_{\left\{\mu \leqslant \exp \left(\frac{\alpha|f|}{2\|f\|_{B M O}}\right)\right\}} \\
\leqslant & \left\{\int_{B} \mu \chi_{\left\{\mu \leqslant \exp \left(\frac{\alpha|f|}{2\|f\|_{B M O}}\right)\right\}}\right\}^{\frac{1}{p}}\left\{\int_{B} \mu|f|^{q} \chi_{\left\{\mu \leqslant \exp \left(\frac{\alpha|f|}{2\|f\| \|_{B M O}}\right)\right\}}\right\}^{\frac{1}{q}}
\end{aligned}
$$

Since $t<\exp (t)$, for all $t \in \mathbb{R}^{+}$, we have $\frac{\alpha|f|}{2 q\|f\|_{B M O}}<\exp \left(\frac{\alpha|f|}{2 q\|f\|_{B M O}}\right)$. Hence, it follows that 


$$
\begin{aligned}
& \left\{\int_{B} \mu|f|^{q} \chi_{\left\{\mu \leqslant \exp \left(\frac{\alpha|f|}{2\|f\|_{B M O}}\right)\right\}}\right\}^{\frac{1}{q}} \\
& \leq \frac{2 q}{\alpha}\|f\|_{B M O}\left\{\int_{B} \exp \left(\frac{\alpha|f|}{2\|f\|_{B M O}}\right)\left[\exp \left(\frac{\alpha|f|}{2 q\|f\|_{B M O}}\right)\right]^{q}\right\}^{\frac{1}{q}} \\
& =\frac{2 q}{\alpha}\|f\|_{B M O}\left\{\int_{B} \exp \left(\frac{\alpha|f|}{2\|f\|_{B M O}}\right) \exp \left(\frac{\alpha|f|}{2\|f\|_{B M O}}\right)\right\}^{\frac{1}{q}} \\
& =\frac{2 q}{\alpha}\|f\|_{B M O}\left\{\int_{B} \exp \left(\frac{\alpha|f|}{\|f\|_{B M O}}\right)\right\}^{\frac{1}{q}} \\
& \leq \frac{2 q}{\alpha}\|f\|_{B M O} K^{\frac{1}{q}}
\end{aligned}
$$

in which we employ Theorem 4.2 due to John and Nirenberg [9] and the condition $\int_{B} f=0$ to deduce the last inequality.

Due to inequalities (4.12) and (4.13), we have

$$
\int_{B} \mu|f| \chi_{\left\{\mu \leqslant \exp \left(\frac{\alpha|f|}{2\|f\|_{B M O}}\right)\right\}} \leqslant\left\{\int_{B} \mu\right\}^{\frac{1}{p}} \frac{2 q}{\alpha}\|f\|_{B M O} K^{1-\frac{1}{p}}
$$

But, on the other hand, we have

$$
\int_{B} \mu|f| \chi_{\left\{\mu>\exp \left(\frac{\alpha|f|}{2\|f\|_{B M O}}\right)\right\}} \leqslant \int_{B} \frac{2}{\alpha}\|f\|_{B M O} \mu \log ^{+} \mu .
$$

By combining inequalities (4.14), and (4.15), we conclude that

$$
\int_{B} \mu|f| \leqslant \frac{2 p}{\alpha(p-1)}\left\{1+K^{1-\frac{1}{p}}\right\}\|f\|_{B M O}\left\{\left(\int_{B} \mu\right)^{\frac{1}{p}}+\int_{B} \mu l o g^{+} \mu\right\}
$$

We are now ready to work with the term $\int_{T_{k-1}}^{1}\left|\int_{\mathbb{R}^{3}} \nabla\left(\frac{v_{k}}{|u|}\right) u P_{k 1} d x\right| d s$.

Indeed, by a simple application of the partial regularity theorem due to Caffarelli, Kohn, and Nirenberg, it can be shown that, if $B$ is a sufficiently large open ball centered at the origin of $\mathbb{R}^{3}$ (we will choose $B$ to be large enough so that it will satisfy $|B|>1$ ), then it follows that the following assertion holds.

- $\left[\frac{1}{2}, 1\right] \times \mathbb{R}^{3} \cap\left\{v_{k} \geqslant 0\right\}$ is a subset of $\left[\frac{1}{2}, 1\right] \times B$, for all $k \geqslant 1$, and if $R$ is sufficiently lage.

Now, let us show the validity of the above assertion more precisely. Here, we will employ the notation $B_{x}(r)=\left\{y \in \mathbb{R}^{3}:|y-x|<r\right\}$. Recall that, the key lemma which leads to the partial regularity theorem of Caffarelli, Kohn, Nirenberg basically asserts that

- there exists a universal constant $\eta^{*}$ such that for any pair of suitable weak solutions $(u, P)$ on $[0,1] \times B_{0}(1)$ satisfying the condition $\|u\|_{L^{3}\left([0,1] \times B_{0}(1)\right)}+$ $\|P\|_{L^{\frac{3}{2}}\left([0,1] \times B_{0}(1)\right)} \leqslant \eta^{*}$, we have $|u| \leqslant 1$ on $\left[\frac{1}{2}, 1\right] \times B_{0}\left(\frac{1}{2}\right)$ (Here, we closely follow the version in [13], see also [3] and [19]).

Since we assume that our suitable weak solution $u:[0,1] \times \mathbb{R}^{3} \rightarrow \mathbb{R}^{3}$ satisfies $u \in$ $L^{\infty}\left(0,1 ; L^{2}\left(\mathbb{R}^{3}\right)\right) \cap L^{2}\left(0,1 ; \dot{H}^{1}\left(\mathbb{R}^{3}\right)\right)$ and $P=\sum R_{i} R_{j} u_{i} u_{j}$, we have $\|u\|_{L^{\frac{10}{3}}\left([0,1] \times \mathbb{R}^{3}\right)}+$ $\|P\|_{L^{\frac{5}{3}\left([0,1] \times \mathbb{R}^{3}\right)}}<\infty$. This indicates that, if we choose the radius $r_{0}>1$ to be sufficiently large, we will have 


$$
\text { - }\|u\|_{L^{\frac{10}{3}}\left([0,1] \times \mathbb{R}^{3}-B_{0}\left(r_{0}\right)\right)}+\|P\|_{L^{\frac{5}{3}\left([0,1] \times \mathbb{R}^{3}-B_{0}\left(r_{0}\right)\right)}}<\frac{\eta^{*}}{|B(1)|^{\frac{2}{30}}} .
$$

So, we can apply the Holder's inequality to deduce that, for any $x \in \mathbb{R}^{3}-B_{0}\left(r_{0}+\right.$ 1), we have

$$
\begin{aligned}
& \|u\|_{L^{3}\left([0,1] \times B_{x}(1)\right)}+\|P\|_{L^{\frac{3}{2}}\left([0,1] \times B_{x}(1)\right)} \\
& \leqslant|B(1)|^{\frac{2}{30}}\left\{\|u\|_{L^{\frac{10}{3}}\left([0,1] \times \mathbb{R}^{3}-B_{0}\left(r_{0}\right)\right)}+\|P\|_{L^{\frac{5}{3}}\left([0,1] \times \mathbb{R}^{3}-B_{0}\left(r_{0}\right)\right)}\right\} \\
& \leqslant \eta^{*}
\end{aligned}
$$

which in turns implies that for any $x \in \mathbb{R}^{3}-B_{0}\left(r_{0}+1\right)$, we have $|u| \leqslant 1$ on $\left[\frac{1}{2}, 1\right] \times B_{x}\left(\frac{1}{2}\right)$. That is, we will have $|u| \leqslant 1$ on $\mathbb{R}^{3}-B_{0}\left(r_{0}+1\right)$. As a result, if we choose $B=B_{0}\left(r_{0}+1\right)$, then it follows that, for each $k \geqslant 1, v_{k}=\left[|u|-R\left(1-\frac{1}{2^{k}}\right)\right]_{+}$ will vanish identically on $\left[\frac{1}{2}, 1\right] \times \mathbb{R}^{3}-B$, for any $R>2$, and hence the validity of the assertion for $B=B_{0}\left(r_{0}+1\right)$ (Here, let us remark that the above idea has also been used in the work [7] of L. Escauriaza, G. Seregin, and V. Sverak ).

On the other hand, since $\nabla\left(\frac{v_{k}}{|u|}\right) u=-R\left(1-\frac{1}{2^{k}}\right) F \chi_{\left\{v_{k}>0\right\}}$. So, we have

$$
\begin{aligned}
\left|\int_{\mathbb{R}^{3}} \nabla\left(\frac{v_{k}}{|u|}\right) u P_{k 1} d x\right| & =\left|\int_{B} R\left(1-\frac{1}{2^{k}}\right) F \chi_{\left\{v_{k}>0\right\}} P_{k 1} d x\right| \\
& \leqslant R \int_{B}|F| \chi_{\left\{v_{k}>0\right\}}\left|P_{k 1}-\left(P_{k 1}\right)_{B}\right| d x \\
& +R \int_{B}|F| \chi_{\left\{v_{k}>0\right\}}\left|\left(P_{k 1}\right)_{B}\right| d x
\end{aligned}
$$

for all $k \geqslant 1$, and all $\frac{1}{2}<t<1$, provided that $R$ is sufficiently large (here, the symbol $\left(P_{k 1}\right)_{B}$ stands for the average value of $P_{k 1}$ over the ball $\left.B\right)$.

Now, since $P_{k 1}=\sum R_{i} R_{j}\left\{\left(1-\frac{w_{k}}{|u|}\right) u_{i}\left(1-\frac{w_{k}}{|u|}\right) u_{j}\right\}$, it follows from the Risez's Theorem in the theory of singular integral that $\left\|P_{k 1}(t, \cdot)\right\|_{L^{2}\left(\mathbb{R}^{3}\right)} \leqslant C_{2} R^{\beta}\|u(t, \cdot)\|_{L^{2}\left(\mathbb{R}^{3}\right)}$, for all $t \in[0,1]$, in which $C_{2}$ is some universal constant. So, we can use the Holder's inequality to carry out the following estimation

$$
\begin{aligned}
\left|\left(P_{k 1}\right)_{B}(t)\right| & \leqslant \frac{1}{|B|} \int_{B}\left|P_{k 1}(t, x)\right| d x \\
& \leqslant \frac{1}{|B|^{\frac{1}{2}}}\left\|P_{k 1}(t, \cdot)\right\|_{L^{2}(B)} \\
& \leqslant \frac{1}{|B|^{\frac{1}{2}}} C_{2} R^{\beta}\|u(t, \cdot)\|_{L^{2}\left(\mathbb{R}^{3}\right)} \\
& \leqslant C_{2} R^{\beta}\|u\|_{L^{\infty}\left(0,1 ; L^{2}\left(\mathbb{R}^{3}\right)\right)} .
\end{aligned}
$$

We remark that the last line of the above inequality holds since our open ball $B$ is sufficiently large so that $|B|>1$. As a result, it follows that

$$
\begin{aligned}
\left|\int_{\mathbb{R}^{3}} \nabla\left(\frac{v_{k}}{|u|}\right) u P_{k 1} d x\right| & \leqslant R \int_{B}|F| \chi_{\left\{v_{k}>0\right\}}\left|P_{k 1}-\left(P_{k 1}\right)_{B}\right| d x \\
& +C_{2} R\|u\|_{L^{\infty}\left(0,1 ; L^{2}\left(\mathbb{R}^{3}\right)\right)} \int_{B} R^{\beta}|F| \chi_{\left\{v_{k}>0\right\}} .
\end{aligned}
$$


Indeed, the operator $R_{i} R_{j}$ is indeed a Zygmund- Calderon operator, and so $R_{i} R_{j}$ must be a bounded operator from $L^{\infty}\left(\mathbb{R}^{3}\right)$ to $B M O\left(\mathbb{R}^{3}\right)$. Hence we can deduce that

$$
\begin{aligned}
\left\|P_{k 1}(t, \cdot)-\left(P_{k 1}\right)_{B}(t)\right\|_{B M O} & =\left\|P_{k 1}(t, \cdot)\right\|_{B M O} \\
& \leqslant C_{0}\left\|\left(1-\frac{w_{k}}{|u|}\right) u_{i}\left(1-\frac{w_{k}}{|u|}\right) u_{j}\right\|_{L^{\infty}\left(\mathbb{R}^{3}\right)} \\
& \leqslant C_{0} R^{2 \beta}
\end{aligned}
$$

for all $t \in(0,1)$, in which $C_{0}$ is some universal constant. So, we now apply Lemma 4.3 with $\mu=|F| \chi_{\left\{v_{k}>0\right\}}$, and $f=P_{k 1}-\left(P_{k 1}\right)_{B}$ to deduce that

$$
\begin{aligned}
\int_{B}|F| \chi_{\left\{v_{k}>0\right\}}\left|P_{k 1}-\left(P_{k 1}\right)_{B}\right| d x & \leqslant \frac{2 p C_{0}}{\alpha(p-1)}\left\{1+K^{1-\frac{1}{p}}\right\} \times \\
& \left\{\left(\int_{B} R^{2 p \beta}|F| \chi_{\left\{v_{k}>0\right\}}\right)^{\frac{1}{p}}+\int_{B} R^{2 \beta}|F| \log ^{+}|F| \chi_{\left\{v_{k}>0\right\}}\right\},
\end{aligned}
$$

in which the symbol $\left(P_{k 1}\right)_{B}$ stands for the mean value of $P_{k 1}$ over the open ball $B$. Since we know that $\left\{v_{k}>0\right\}$ is a subset of $\left\{|u|>\frac{R}{2}\right\}$, for all $k \geqslant 1$, so it follows from the above inequality that

$$
\begin{aligned}
\int_{B}|F| \chi_{\left\{v_{k}>0\right\}}\left|P_{k 1}-\left(P_{k 1}\right)_{B}\right| d x & \leqslant \frac{2 C_{0}}{\alpha} \frac{p}{p-1} 4^{p \beta}\left\{1+K^{1-\frac{1}{p}}\right\} \times \\
& \left\{\left(\int_{B}|u|^{2 p \beta}|F| \chi_{\left\{v_{k}>0\right\}}\right)^{\frac{1}{p}}\right. \\
& \left.+\int_{B}|u|^{2 \beta}|F| \log ^{+}|F| \cdot \chi_{\left\{v_{k}>0\right\}}\right\} .
\end{aligned}
$$

So, we can conclude from inequality (4.17), and the above inequality that

$$
\begin{aligned}
\int_{T_{k-1}}^{1}\left|\int_{\mathbb{R}^{3}} \nabla\left(\frac{v_{k}}{|u|}\right) u P_{k 1} d x\right| d t & \leqslant R \frac{2 C_{0}}{\alpha} \frac{p}{p-1} 4^{p \beta}\left(1+K^{1-\frac{1}{p}}\right) \times \\
& \left\{\left(\int_{Q_{k-1}}|u|^{2 p \beta}|F| \chi_{\left\{v_{k}>0\right\}}\right)^{\frac{1}{p}}\right. \\
& \left.+\int_{Q_{k-1}}|u|^{2 \beta}|F| \log (1+|F|) \chi_{\left\{v_{k}>0\right\}}\right\} \\
& +C_{2} 2^{\beta} R\|u\|_{L^{\infty}\left(0,1 ; L^{2}\left(\mathbb{R}^{3}\right)\right)} \int_{Q_{k-1}}|u|^{\beta}|F| \chi_{\left\{v_{k}>0\right\}} .
\end{aligned}
$$

Now, notice that

$$
\begin{aligned}
\int_{Q_{k-1}}|u|^{2 \beta}|F| \log (1+|F|) \chi_{\left\{v_{k}>0\right\}} & \leqslant \int_{Q_{k-1}}|u|^{2 \beta}|F| \log (1+|F|) \chi_{\left\{|F| \leqslant \frac{1}{R}\right\}} \chi_{\left\{v_{k}>0\right\}} \\
& +\int_{Q_{k-1}}|u|^{2 \beta}|F| \log (1+|F|) \chi_{\left\{|F|>\frac{1}{R}\right\}} \chi_{\left\{v_{k}>0\right\}} \\
& \leqslant \frac{\log 2}{R} \int_{Q_{k-1}}|u|^{2 \beta} \chi_{\left\{v_{k}>0\right\}} \\
& +\int_{Q_{k-1}}|u|^{2 \beta}|F| \log (1+|F|) \chi_{\left\{|F|>\frac{1}{R}\right\}} \chi_{\left\{v_{k}>0\right\}}
\end{aligned}
$$


Step five. To deal with the second term in the last line of inequality (4.19), we consider the sequence $\left\{\phi_{k}\right\}_{k=1}^{\infty}$ of nonnegative continuous functions on $[0, \infty)$, which are defined by

- $\phi_{k}(t)=0$, for all $t \in\left[0, C_{k}\right]$.

- $\phi_{k}(t)=t-C_{k}$, for all $t \in\left(C_{k}, C_{k}+1\right)$.

- $\phi_{k}(t)=1$, for all $t \in\left[C_{k}+1,+\infty\right)$.

where the symbol $C_{k}$ stands for $C_{k}=R\left(1-\frac{1}{2^{k}}\right)$, for every $k \geqslant 1$. Here, we remark that, for the purpose of taking spatial derivative, the composite function $\phi_{k}(|u|)$ is a good substitute for $\chi_{\left\{v_{k}>0\right\}}=\chi_{\left\{|u|>R\left(1-\frac{1}{2^{k}}\right)\right\}}$, since $\phi_{k}$ is Lipschitz.

Moreover, we also need a smooth function $\psi: \mathbb{R} \rightarrow \mathbb{R}$ satisfying the following conditions that:

- $\psi(t)=1$, for all $t \geqslant \frac{1}{R}$.

- $0<\psi(t)<1$, for all $t$ with $0<t<\frac{1}{R}$.

- $\psi(0)=0$.

- $-1<\psi(t)<0$, for all $t$ with $-\frac{1}{R}<t<0$.

- $\psi(t)=-1$, for all $t \leqslant-\frac{1}{R}$.

- $0 \leqslant \frac{d}{d t} \psi \leqslant 2 R$, for all $t \in \mathbb{R}$.

We further remark that the smooth function $\psi: \mathbb{R} \rightarrow \mathbb{R}$ characterized by the above properties must also satisfy the property that $\frac{d \psi}{d t}(t)=0$, on $t \in\left(-\infty,-\frac{1}{R}\right) \cup$ $\left(\frac{1}{R}, \infty\right)$, which will be employed in forthcoming inequality estimations (4.21) and (4.24) without explicit mention. With the above preperation, let $\beta$ be such that $3<2 \beta<\frac{10}{3}+1-\gamma$. We can then carry out the following calculation

$$
\begin{aligned}
\operatorname{div}\left\{|u|^{2 \beta-1} u \psi(F) \log (1+|F|) \phi_{k}(|u|)\right\} & =-(2 \beta-1)|u|^{2 \beta} F \psi(F) \log (1+|F|) \phi_{k}(|u|) \\
& -|u|^{2 \beta+1} F \psi(F) \log (1+|F|) \chi_{\left\{C_{k}<|u|<C_{k}+1\right\}} \\
& +|u|^{2 \beta-1} \frac{d \psi}{d t}(F)(u \cdot \nabla F) \log (1+|F|) \phi_{k}(|u|) \\
& +|u|^{2 \beta-1} \psi(F) \frac{u \cdot \nabla|F|}{1+|F|} \phi_{k}(|u|) .
\end{aligned}
$$

Since our weak solution $u$ on $(0,1] \times \mathbb{R}^{3}$ satisfies $\frac{|u \cdot \nabla F|}{|u|^{\gamma}} \leqslant A|F|$, it follows that

- $|u \cdot \nabla F|(t, x) \leqslant \frac{A}{R}|u(t, x)|^{\gamma}$, if it happens that $(t, x)$ satisfies $|F(t, x)| \leqslant \frac{1}{R}$.

- $\left|\frac{u \cdot \nabla|F|}{1+|F|}\right| \leqslant \frac{|u \cdot \nabla| F||}{|F|}=\frac{|u \cdot \nabla F|}{|F|} \leqslant A|u|^{\gamma}$.

So, it follows from inequality (4.20) that 


$$
\begin{aligned}
\Lambda_{1}+\Lambda_{2} & \leqslant \int_{Q_{k-1}}|u|^{2 \beta-1}\left|\frac{d \psi}{d t}(F)\right| \cdot|u \cdot \nabla F| \log (1+|F|) \phi_{k}(|u|) \\
& +\int_{Q_{k-1}}|u|^{2 \beta-1}|\psi(F)| \cdot\left|\frac{u \cdot \nabla|F|}{1+|F|}\right| \phi_{k}(|u|) \\
& \leqslant \int_{Q_{k-1}}|u|^{2 \beta-1}(2 R)\left(\frac{A}{R}|u|^{\gamma}\right) \log \left(1+\frac{1}{R}\right) \phi_{k}(|u|) \\
& +\int_{Q_{k-1}}|u|^{2 \beta-1} \cdot A \cdot|u|^{\gamma} \phi_{k}(|u|) \\
& \leqslant A(1+2 \log 2) \int_{Q_{k-1}}|u|^{2 \beta-1+\gamma} \phi_{k}(|u|) \\
& \leqslant A(1+2 \log 2) \int_{Q_{k-1}}|u|^{2 \beta-1+\gamma} \chi_{\left\{v_{k}>0\right\}},
\end{aligned}
$$

in which the terms $\Lambda_{1}$, and $\Lambda_{2}$ are given by

- $\Lambda_{1}=(2 \beta-1) \int_{Q_{k-1}}|u|^{2 \beta} F \psi(F) \cdot \log (1+|F|) \phi_{k}(|u|)$.

- $\Lambda_{2}=\int_{Q_{k-1}}|u|^{2 \beta+1}(\psi(F) F) \cdot \log (1+|F|) \chi_{\left\{C_{k}<|u|<C_{k}+1\right\}}$.

We then notice that

- Since $2 \beta>3>2$, we have $\Lambda_{1} \geqslant \int_{Q_{k-1}}|u|^{2 \beta}(F \psi(F)) \log (1+|F|) \chi_{\left\{|u| \geqslant C_{k}+1\right\}}$.

- $\Lambda_{2} \geqslant \frac{R}{2} \int_{Q_{k-1}}|u|^{2 \beta} F \psi(F) \log (1+|F|) \chi_{\left\{C_{k}<|u|<C_{k}+1\right\}}$, for every $k \geqslant 1$. Notice that this is true because $C_{k}=R\left(1-\frac{1}{2^{k}}\right)$, and that $\left(1-\frac{1}{2^{k}}\right) \geqslant \frac{1}{2}$, for every $k \geqslant 1$.

Hence, it follows from inequality (4.21) that

$$
\begin{aligned}
& \int_{Q_{k-1}}|u|^{2 \beta} F \psi(F) \log (1+|F|) \chi_{\left\{v_{k}>0\right\}} \\
& =\int_{Q_{k-1}}|u|^{2 \beta} F \psi(F) \log (1+|F|) \chi_{\left\{C_{k}<|u|<C_{k}+1\right\}} \\
& +\int_{Q_{k-1}}|u|^{2 \beta} F \psi(F) \log (1+|F|) \chi_{\left\{|u| \geqslant C_{k}+1\right\}} \\
& \leqslant \frac{2}{R} \Lambda_{2}+\Lambda_{1} \\
& \leqslant 3 C \cdot A \int_{Q_{k-1}}|u|^{2 \beta-1+\gamma} \chi_{\left\{v_{k}>0\right\}} .
\end{aligned}
$$

As a matter of fact, inequality (4.22) leads us to raise up the index for the term $\int_{Q_{k-1}}|u|^{\theta} \chi_{\left\{v_{k}>0\right\}}$, for any $\theta$ with $0<\theta<\frac{10}{3}$, in the following way

$$
\begin{aligned}
\int_{Q_{k-1}}|u|^{\theta} \chi_{\left\{v_{k}>0\right\}} & =\int_{Q_{k-1}}\left\{R\left(1-\frac{1}{2^{k}}\right)+v_{k}\right\}^{\theta} \chi_{\left\{v_{k}>0\right\}} \\
& \leqslant C_{\theta}\left\{R^{\theta} \int_{Q_{k-1}} \chi_{\left\{v_{k}>0\right\}}+\int_{Q_{k-1}} v_{k}^{\theta} \chi_{\left\{v_{k}>0\right\}}\right\} \\
& \leqslant \frac{C_{\theta}}{R^{\frac{10}{3}-\theta}}\left\{2^{\frac{10 k}{3}}+2^{\left(\frac{10}{3}-\theta\right) k}\right\} \int_{Q_{k-1}} v_{k-1}^{\frac{10}{3}} \\
& \leqslant \frac{C_{\theta}}{R^{\frac{10}{3}-\theta}} 2^{\frac{10 k}{3}} U_{k-1}^{\frac{5}{3}}
\end{aligned}
$$


for every $\theta$ with $0<\theta<\frac{10}{3}$, where $C_{\theta}$ is some positive constant depending only on $\theta$. Hence it follows from inequalities(4.19), (4.22), and our last inequality that

$$
\begin{aligned}
\int_{Q_{k-1}}|u|^{2 \beta}|F| \cdot \log (1+|F|) \chi_{\left\{v_{k}>0\right\}} & \leqslant \frac{\log 2}{R} \int_{Q_{k-1}}|u|^{2 \beta} \chi_{\left\{v_{k}>0\right\}} \\
& +\int_{Q_{k-1}}|u|^{2 \beta}|F| \log (1+|F|) \chi_{\left\{|F|>\frac{1}{R}\right\}} \chi_{\left\{v_{k}>0\right\}} \\
& \leqslant \frac{\log 2}{R} \frac{C_{2 \beta} 2^{\frac{10 k}{3}}}{R^{\frac{10}{3}-2 \beta}} U_{k-1}^{\frac{5}{3}} \\
& +3 C \cdot A \int_{Q_{k-1}}|u|^{2 \beta-1+\gamma} \chi_{\left\{v_{k}>0\right\}} \\
& \leqslant C_{\beta, \gamma}(1+A) \cdot 2^{\frac{10 k}{3}} U_{k-1}^{\frac{5}{3}}\left\{\frac{1}{R^{\frac{10}{3}-2 \beta+1}}\right. \\
& \left.+\frac{1}{R^{\frac{10}{3}-2 \beta+1-\gamma}}\right\}
\end{aligned}
$$

in which $\beta>\frac{3}{2}$, and that $\beta$ is sufficiently close to $\frac{3}{2}$, and $C_{\beta, \gamma}$ is some constant depending only on $\beta$, and $\gamma$.

Next, we also need to deal with $\left(\int_{Q_{k-1}}|u|^{2 p \beta}|F| \chi_{\left\{v_{k} \geqslant 0\right\}}\right)^{\frac{1}{p}}$, and $\int_{Q_{k-1}}|u|^{\beta}|F| \chi_{\left\{v_{k} \geqslant 0\right\}}$, which appear in inequality (4.18). For this purpose, we will consider $\lambda$ which satisfies $\frac{3}{2}<\lambda<\frac{10}{3}+1-\gamma$ (we will take $\lambda$ to be $2 p \beta$ and $\beta$ respectively in forthcoming inequality estimates (4.25) and (4.26)), and let us carry out the following computation, in which $\psi$ and $\phi_{k}$ etc are just the same as before.

$$
\begin{aligned}
\operatorname{div}\left\{|u|^{\lambda-1} u \psi(F) \phi_{k}(|u|)\right\} & =-(\lambda-1)|u|^{\lambda} F \psi(F) \phi_{k}(|u|) \\
& +|u|^{\lambda-1} \frac{d \psi}{d t}(F)(u \cdot \nabla F) \phi_{k}(|u|) \\
& -|u|^{\lambda+1} F \psi(F) \chi_{\left\{C_{k}<|u|<C_{k}+1\right\}} .
\end{aligned}
$$

Hence, we have

$$
\begin{aligned}
(\lambda-1) \int_{Q_{k-1}}|u|^{\lambda} F \psi(F) \phi_{k}(|u|) & +\int_{Q_{k-1}}|u|^{\lambda+1} F \psi(F) \chi_{\left\{C_{k}<|u|<C_{k}+1\right\}} \\
& \leqslant \int_{Q_{k-1}}|u|^{\lambda-1}\left|\frac{d \psi}{d t}(F)\right| \cdot|u \cdot \nabla F| \phi_{k}(|u|) \\
& \leqslant \int_{Q_{k-1}}|u|^{\lambda-1}(2 R)\left(\frac{A}{R}|u|^{\gamma}\right) \chi_{\left\{v_{k}>0\right\}} \\
& \leqslant 2 A \int_{Q_{k-1}}|u|^{\lambda-1+\gamma} \chi_{\left\{v_{k}>0\right\}} .
\end{aligned}
$$


By the same calculation as in inequality (4.21), we can see that

$$
\begin{aligned}
\int_{Q_{k-1}}|u|^{\lambda} F \psi(F) \chi_{\left\{v_{k}>0\right\}} & =\int_{Q_{k-1}}|u|^{\lambda} F \psi(F) \chi_{\left\{C_{k}<|u|<C_{k}+1\right\}} \\
& +\int_{Q_{k-1}}|u|^{\lambda} F \psi(F) \chi_{\left\{|u| \geqslant C_{k}+1\right\}} \\
& \leqslant \frac{2}{R} \int_{Q_{k-1}}|u|^{\lambda+1} F \psi(F) \chi_{\left\{C_{k}<|u|<C_{k}+1\right\}} \\
& +\int_{Q_{k-1}}|u|^{\lambda} F \psi(F) \phi_{k}(|u|) \\
& \leqslant 3\left\{\int_{Q_{k-1}}|u|^{\lambda+1} F \psi(F) \chi_{\left\{C_{k}<|u|<C_{k}+1\right\}}\right. \\
& \left.+(\lambda-1) \int_{Q_{k-1}}|u|^{\lambda} F \psi(F) \phi_{k}(|u|)\right\} \\
& \leqslant 6 A \int_{Q_{k-1}}|u|^{\lambda-1+\gamma} \chi_{\left\{v_{k}>0\right\}},
\end{aligned}
$$

in which $\lambda$ satisfies $\frac{3}{2}<\lambda<\frac{10}{3}+1-\gamma$. Now, put $\lambda=2 p \beta$, with $\beta>\frac{3}{2}$ to be sufficiently close to $\frac{3}{2}$, and $1<p<\frac{5}{4}$ to be sufficiently close to 1 , it follows from our last inequality that

$$
\begin{aligned}
\int_{Q_{k-1}}|u|^{2 p \beta}|F| \chi_{\left\{v_{k}>0\right\}} & =\int_{Q_{k-1}}|u|^{2 p \beta}|F| \chi_{\left\{|F| \leqslant \frac{1}{R}\right\}} \chi_{\left\{v_{k}>0\right\}} \\
& +\int_{Q_{k-1}}|u|^{2 p \beta} \chi_{\left\{|F|>\frac{1}{R}\right\}} \chi_{\left\{v_{k}>0\right\}}|F| \\
& \leqslant \frac{1}{R} \int_{Q_{k-1}}|u|^{2 p \beta} \chi_{\left\{v_{k}>0\right\}} \\
& +6 A \int_{Q_{k-1}}|u|^{2 p \beta-1+\gamma} \chi_{\left\{v_{k}>0\right\}} \\
& \leqslant C(1+A)\left\{\frac{1}{R^{\frac{10}{3}-2 p \beta+1}}+\frac{1}{\left.R^{\frac{10}{3}-2 p \beta+1-\gamma}\right\} 2^{\frac{10 k}{3}} U_{k-1}^{\frac{5}{3}} .}\right.
\end{aligned}
$$

In exactly the same way, by setting $\lambda$ to be $\beta$, with $\beta>\frac{3}{2}$ to be sufficiently close to $\frac{3}{2}$, it also follows that

$$
\begin{aligned}
\int_{Q_{k-1}}|u|^{\beta}|F| \chi_{\left\{v_{k}>0\right\}} & =\int_{Q_{k-1}}|u|^{\beta}|F| \chi_{\left\{|F| \leqslant \frac{1}{R}\right\}} \chi_{\left\{v_{k}>0\right\}} \\
& +\int_{Q_{k-1}}|u|^{\beta}|F| \chi_{\left\{|F|>\frac{1}{R}\right\}} \chi_{\left\{v_{k}>0\right\}} \\
& \leqslant \frac{1}{R} \int_{Q_{k-1}}|u|^{\beta} \chi_{\left\{v_{k}>0\right\}}+6 A \int_{Q_{k-1}}|u|^{\beta-1+\gamma} \chi_{\left\{v_{k}>0\right\}} \\
& \leqslant C_{\beta, \gamma}(1+A)\left\{\frac{1}{R^{\frac{10}{3}-\beta+1}}+\frac{1}{R^{\frac{10}{3}-\beta+1-\gamma}}\right\} 2^{\frac{10 k}{3}} U_{k-1}^{\frac{5}{3}}
\end{aligned}
$$



that

By combining inequalities (4.18), (4.23), and (4.25), and (4.26) we now conclude

$$
\begin{aligned}
& \int_{T_{k-1}}^{1}\left|\int_{\mathbb{R}^{3}} \nabla\left(\frac{v_{k}}{|u|}\right) u P_{k 1} d x\right| d s \\
& \leqslant(1+A)\left(1+\frac{1}{\alpha}\right) C_{p, \beta}\left(1+K^{1-\frac{1}{p}}\right) \times \\
& \left(1+\|u\|_{L^{\infty}\left(0,1 ; L^{2}\left(\mathbb{R}^{3}\right)\right)}\right) \times \\
& \left\{\left(\frac{1}{R^{\frac{10}{3}-2 p \beta+1-\gamma-p}}\right)^{\frac{1}{p}} 2^{\frac{10 k}{3 p}} U_{k-1}^{\frac{5}{3 p}}+\frac{1}{R^{\frac{10}{3}-2 \beta-\gamma}} 2^{\frac{10 k}{3}} U_{k-1}^{\frac{5}{3}}\right\} .
\end{aligned}
$$

Notice that if $p \rightarrow 1^{+}$, and $\beta \rightarrow \frac{3}{2}^{+}$, then,we have $\left(\frac{10}{3}-2 p \beta+1-p-\gamma\right) \rightarrow$ $\left(\frac{1}{3}-\gamma\right)>0$, and that $\left(\frac{10}{3}-2 \beta-\gamma\right) \rightarrow\left(\frac{1}{3}-\gamma\right)>0$.

So, finally, we recognize that by combining inequalities (4.10), (4.11), and (4.27), we conclude that we are done in proving proposition 2.1 .

\section{REFERENCES}

[1] J. T. BeAle, T. KATO, AND A. MAJDA, Remarks on the breakdown of smooth solutions for the 3-D Euler equations, Comm. Math. Phys., 94:1 (1984), pp. 61-66.

[2] H. Beirao DA Veiga, A new regularity class for the Navier-Stokes equations in $\mathbb{R}^{n}$, Chinese Ann. Math. Ser. B, 16:4 (1995), pp. 407-412. A Chinese summary appears in Chinese Ann. Math. Ser. A, 16:6 (1995), p. 797.

[3] L. Caffarelli, R. Kohn, and L. Nirenberg, Partial regularity of suitable weak solutions of the Navier-Stokes equations, Comm. Pure Appl. Math., 35:6 (1982), pp. 771-831.

[4] Ch.-H. Chan and A. Vasseur, Log improvement of the Prodi-Serrin criteria for Navier-Stokes equation, Methods Appl. Anal., 14:2 (2007), pp. 197-212.

[5] P. Constantin and C. Fefferman, Direction of vorticity and the problem of global regularity for the Navier-Stokes equations, Indiana Univ. Math. J., 42:3 (1993), pp. 775-789.

[6] E. De Giongi, Sulla differenziabilita e l'analiticita delle estremali degli integrali multipli regolari, Mem. Accad. Sci. Torino. Cl. Sci. Fis. Mat. Nat. (3), 3 (1957), pp. 25-43.

[7] L. Escauriaza, G. Seregin, And V. Sverak, $L_{3, \infty}$-solutions of the Navier-Stokes equations and backward uniqueness, Russian Math. Surveys, 58:2 (2003), pp. 211-250.

[8] E. Hopf, Uber die Anfangswertaufgabe fur die hydrodynamischen Grundgleichungen, Math. Nachr., 4 (1951), pp. 213-231.

[9] F. John And L. Nirenberg, On functions of bounded mean oscillation, Comm. Pure Appl. Math., 14 (1961), pp. 415-426.

[10] H. Kozono AND Y. TANiUCHI, Bilinear estimates in BMO and the Navier-Stokes equations, math. Z., 235 (2000), pp. 173-194.

[11] I. Kukavica AND M. Ziane, Navier-Stokes equations with regularity in one direction, J. Math. Phys., 48:6 (2007), 065203.

[12] J. LeRAY, Sur le mouvement d'un liquide visqueux emplissant l'espace, Acta. Math., 63 (1934), pp. $183-248$.

[13] F. Lin, A new proof of the Caffarelli-Kohn-Nirenberg theorem, Comm. Pure Appl. Math., 51:3 (1998), pp. 241-257.

[14] G. Prodi, Un teorema di unicita per le equazioni di Navier-Stokes, Ann. Mat. Pura Appl. (4), 48 (1959), pp. 173-182.

[15] G. Seregin And V. Sverak, Navier-Stokes equations with lower bounds on the pressure, Arch. Ration. Mech. Anal., 163:1 (2002), pp. 65-86.

[16] J. SERRIN, The initial value problem for the Navier-Stokes equations, in "Nonlinear Problems Proc. Sympos., Madison, Wis.”, pp. 69-98. Univ. of Wisconsin Press, Madison, Wis., 1963.

[17] M. Struwe, On partial regularity results for the Navier-Stokes equations, Comm. Pure Appl. Math., 41:4 (1988), pp. 437-458.

[18] G. Tian And Z. XIN, Gradient estimation on Navier-Stokes equations, Comm. Anal. Geom., 7:2 (1999), pp. 221-257.

[19] A. VASSEUR, A new proof of partial regularity of solutions to Navier-Stokes equations, NoDEA Nonlinear Differential Equations Appl., 14:5-6 (2007), pp. 753-785. 
[20] A. VAsSEuR, Regularity criterion for 3D Navier-Stokes equations in terms of the direction of the velocity, Appl. Math., 54:1 (2009), pp. 47-52.

[21] Y. ZHOU, A new regularity criterion for weak solutions to the Navier-Stokes equations, J. Math. Pures Appl., (9) 84:11 (2005), pp. 1496-1514.

[22] Y. ZHOU, A new regularity criterion for the Navier-Stokes equations in terms of the gradient of one velocity component, Methods Appl. Anal., 9:4 (2002), pp. 563-578.

[23] Y. ZHOU AND M. POKORNY, On a regularity criterion for the Navier Stokes equations involving gradient of one velocity component, J. Math. Phys., 50 (2009), 123514.

[24] Y. ZHOU, Direction of vorticity and a new regularity criterion for the Navier-Stokes equations, ANZIAM J., 46:3 (2005), pp. 309-316.

[25] Y. Zhou, A new regularity criterion for the Navier-Stokes equations in terms of the direction of vorticity, Monatsh. Math., 144:3 (2005), pp. 251-257.

[26] Y. ZHou, On regularity criteria in terms of pressure for the Navier-Stokes equations in $\mathbb{R}^{3}$, Proc. Amer. Math. Soc., 134:1 (2006), pp. 149-156.

[27] Y. ZHOU, On a regularity criterion in terms of the gradient of pressure for the Navier-Stokes equations in $\mathbb{R}^{N}$, Z. Angew. Math. Phys., 57:3 (2006), pp. 384-392. 
C. H. CHAN 